
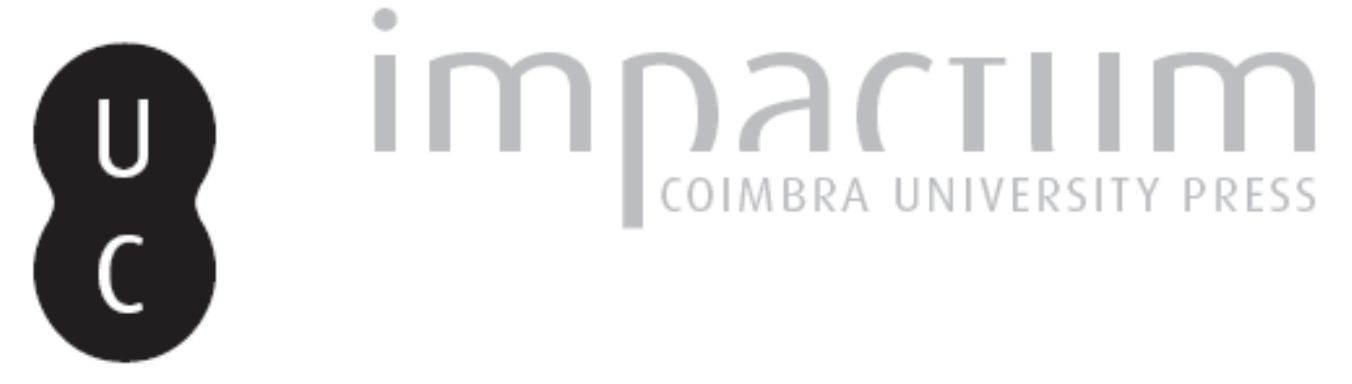

\title{
Les divinités indigènes de Vettonnie sous le Haut-Empire romain: essai d'inventaire et interprétation
}

Autor(es): Bonnaud, Christophe

Publicado por: Faculdade de Letras da Universidade de Coimbra

URL persistente:

URI:http://hdl.handle.net/10316.2/37665

DOI:

DOI:http://dx.doi.org/10.14195/1647-8657_41_3

Accessed : $\quad$ 26-Apr-2023 09:23:43

A navegação consulta e descarregamento dos títulos inseridos nas Bibliotecas Digitais UC Digitalis, UC Pombalina e UC Impactum, pressupõem a aceitação plena e sem reservas dos Termos e Condições de Uso destas Bibliotecas Digitais, disponíveis em https://digitalis.uc.pt/pt-pt/termos.

Conforme exposto nos referidos Termos e Condições de Uso, o descarregamento de títulos de acesso restrito requer uma licença válida de autorização devendo o utilizador aceder ao(s) documento(s) a partir de um endereço de IP da instituição detentora da supramencionada licença.

Ao utilizador é apenas permitido o descarregamento para uso pessoal, pelo que o emprego do(s) título(s) descarregado(s) para outro fim, designadamente comercial, carece de autorização do respetivo autor ou editor da obra.

Na medida em que todas as obras da UC Digitalis se encontram protegidas pelo Código do Direito de Autor e Direitos Conexos e demais legislação aplicável, toda a cópia, parcial ou total, deste documento, nos casos em que é legalmente admitida, deverá conter ou fazer-se acompanhar por este aviso.

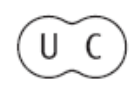




\section{CONIMBRIGA}

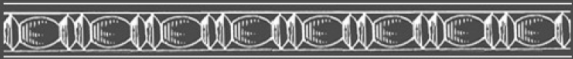

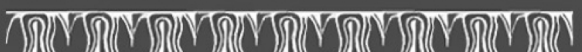
2

INSTITUTO DE ARQUEOLOGIA

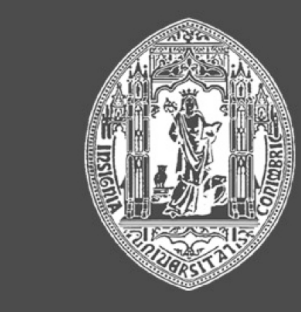

VOLUME XLI - 2002

F A C U L D A E D E LETRAS UNIVERSIDADE DE COIMBRA 
Christophe BonNAud

Docteur en Histoire Ancienne

Professeur d'histoire au Lycée Français du Caire (Egypte)

LES DIVINITÉS INDIGÈNES DE VETTONNIE SOUS LE HAUT-EMPIRE ROMAIN: ESSAI D'INVENTAIRE ET INTERPRÉTATION

"Conimbriga" XLI (2002) p. 63-103

RESUME: $\quad$ Sous le Haut-Empire romain, les Vettones étaient intégrés dans la province de Lusitanie et se situaient plus précisément dans la partie orientale du conventus de Mérida. Au sein de la documentation épigraphique de cette région, nous disposons d'un nombre considérable d'autels votifs, pour la plupart datés des trois premiers siècles de notre ère, qui nous livrent de nombreux théonymes indigènes, certains à l'orthographe incertaine du fait des problèmes de transcription, attestant par là-même la survie de cultes qui trouvent leur origine dans la période préromaine. Nous essayerons de procéder à un inventaire de ces documents épigraphiques qui proviennent de ce que nous croyons être le territoire vetton, c'est-à-dire une grande partie des provinces espagnoles de Salamanque, Ávila, Cáceres, débordant sur celles de Tolède et Badajoz mais aussi une partie du Portugal (à l'est de la serra da Estrela). Il s'agira également de tenter de cerner ce qui fait la particularité de ce panthéon, tant en ce qui concerne la nature et la fonction des divinités adorées que la localisation des autels votifs, ainsi que le milieu social qui pratiquait ces cultes. Cette recherche s'insère dans une étude plus globale sur les pratiques religieuses des Vettons sous le Haut-Empire romain.

RESUMO: As divindades indígenas da Vetónia no Alto Império romano: ensaio de inventário e interpretação

Durante o Alto Império romano, os Vettones estavam integrados na Lusitânia e situavam-se, mais precisamente, na parte oriental do conventus de Mérida. 
A documentação epigráfica dessa região facultou-nos um número considerável de aras votivas, datadas na sua maior parte dos primeiros três séculos da nossa era, com numerosos teónimos indígenas, alguns deles de grafia incerta devido a problemas de transcrição, mas que atestam, mesmo assim, a sobrevivência de cultos que remontam à época pré-romana.

Procuraremos elaborar o inventário dos documentos que pensamos serem provenientes do território vetão, isto é, duma região que compreende grande parte das províncias espanholas de Salamanca, Ávila e Cáceres, atingindo mesmo as de Toledo e Badajoz, assim como uma parte de Portugal (a nascente da Serra da Estrela).

Tentar-se-á, por outro lado, discernir o que distingue este panteão quer no que concerne à natureza e função das divindades adoradas quer no que respeita à localização das aras e, também, ao meio social em que se movimentavam os fiéis desses cultos.

Esta pesquisa insere-se num estudo mais vasto acerca das práticas religiosas dos Vetões durante o Alto Império romano. 


\section{LES DIVINITÉS INDIGÈNES DE VETTONNIE SOUS LE HAUT-EMPIRE ROMAIN: ESSAI D'INVENTAIRE ET INTERPRÉTATION}

L'étude de la religion païenne s'est toujours révélée extrêmement fructueuse en ce qui concerne la péninsule ibérique dans l'Antiquité. L'Hispania est en effet à juste titre considérée comme un domaine géographique où les cultes indigènes ont connu une étonnante profusion et où l'influence religieuse des peuples conquérants, carthaginois mais surtout romains, s'est diffusée y compris jusqu'aux territoires les plus isolés ${ }^{1}$.

L'intégration plus directe de la péninsule au monde romain, liée à son occupation définitive au début de l'Empire, a ensuite redonné vigueur à la religion romaine, avec notamment le développement du culte impérial, sans que l'on puisse jamais parler, de la part des Romains, d'une politique religieuse anti-indigène, bien au contraire ${ }^{2}$.

A ces données très générales, il est cependant nécessaire d'ajouter un élément primordial dans l'étude des pratiques religieuses dans la péninsule ibérique romaine: l'ensemble des peuples ibériques, et au sein de chacun d'entre eux l'ensemble de la société, n'ont pas réagi unanimement et également face au nouveau panthéon, face à de nouvelles coutumes religieuses. Ainsi, comme l'a écrit L. A. Curchin, il y eut une tendance, en particulier dans les zones les plus romanisées de la péninsule, à adopter le culte des dieux romains, ou bien à modifier une divinité

1 Pour une première approche générale, cf. J. BEAUJEU, Cultes locaux et cultes d'Empire dans les provinces d'Occident aux trois premiers siècles de notre ère, dans Assimilation et résistance à la culture gréco-romaine dans le monde ancien. VIème Congrès International d'Etudes Classiques (Madrid, 1974), Paris, 1976, pp. 433-443 (= BeAujeu, Cultes locaux).

2 A. Tranoy, Histoire religieuse, dans R. Etienne et F. Mayet (éd.), Histoire et archéologie de la péninsule ibérique antique. Chroniques quinquennales, 1968-1987, Paris, 1993, p. 407. 
indigène de façon syncrétique et à l'assimiler à un dieu romain ${ }^{3}$. D'autres régions, plus occidentales et plus septentrionales, plus tardivement rattachées à la domination romaine, sont restées plus proches de leurs pratiques du temps de l'indépendance, tout en connaissant ce syncrétisme. Toutefois, on ne peut en aucun cas y évoquer l'absence d'influence romaine dans le domaine religieux: la pratique même de l'épigraphie, funéraire ou votive, est la première manifestation de cet apport romain, faisant ainsi sortir de leur anonymat des centaines de noms de divinités.

En outre, et comme l'a rappelé $\mathrm{A}$. Tranoy, on peut s'interroger à juste titre sur la façon dont les cultes introduits par les conquérants furent compris par les indigènes ${ }^{4}$. Nous touchons là au délicat problème des mentalités religieuses, un domaine encore très mal connu et qui constitue, outre les problèmes de transcription, un obstacle de poids à l'étude des cultes dans la péninsule ibérique sous la domination romaine. Ainsi, comment expliquer la démarche spirituelle (ou de mode?) d'un indigène qui élève un autel à une divinité romaine alors que rien ne semble justifier sur le document épigraphique lui-même ou dans le contexte archéologique environnant, cette dévotion? De même, comment interpréter le fait que la plus grande partie des inscriptions dédiées à Jupiter se rencontrent dans l'ouest de la péninsule ${ }^{5}$ ?

Ces problèmes généraux se retrouvent dans l'étude des pratiques religieuses des Vettons sous la domination romaine: problèmes de transcription, de syncrétisme, d'interprétation... Néanmoins, et bien que nombre de théonymes soient encore peu fiables et qu'il soit souvent difficile de nettement différencier sentiment religieux véritable et phénomène de mode, nous essayerons de parvenir à une vision globale et actualisée au vu des derniers travaux, des croyances et cultes des Vettons sous la domination romaine. Dans un premier temps, nous nous intéresserons aux cultes indigènes, ce sera l'objet de cet article, puis aux divinités classiques; enfin, le thème de la mort devrait nous permettre d'éclaircir un autre aspect des croyances des Vettons.

La finalité d'une telle approche est de mieux cerner la place de la religion vettonne au sein de l'ensemble péninsulaire et notamment vis-

3 L. A. Curchin, Roman Spain. Conquest and assimilation, Londres et New York, 1991, p. 154.

4 A. Tranoy, La Galice romaine. Recherches sur le nord-ouest de la péninsule ibérique dans l'Antiquité, Paris, 1981, p. 264 (= Tranoy, Galice).

5 Tranoy, Galice, p. 415. 
à-vis des peuples voisins des Vettons ${ }^{6}$. Peut-on affirmer, à la suite de J. $\mathrm{J}$. Sayas et de R. López que les "données à caractère religieux que nous possédons sur les Vettons sont généralisables dans leur immense majorité à d'autres peuples péninsulaires" 7 ? Cette opinion ne reposet-elle pas sur une interprétation incomplète et parfois erronée des témoignages archéologiques et épigraphiques, sur une prise en considération insuffisante de ce qu'est vraiment la Vettonnie? Il nous semble important de rappeler qu'on ne peut guère séparer croyances religieuses et traits culturels d'un territoire, ces derniers se reflétant également dans le type de développement économique et d'organisation sociale. Or, à une Vettonnie septentrionale, peu fertile et très peu urbanisée s'opposait une Vettonnie plus méridionale, aux terres moins ingrates et aux traits moins marqués par l'indigénisme, davantage et plus facilement assimilée par le monde romain. Il s'agira de voir si la religion répond également à ce schéma, et s'il existe des noyaux de tradition religieuse préromaine, ou bien au contraire si le fait religieux transcende cette opposition. A l'intérieur des trois thèmes précédemment cités, notre démarche sera donc à nouveau géographique. Mais au préalable, il nous semble nécessaire de revenir rapidement sur l'identité vettonne.

Dans l'Antiquité romaine, la partie orientale du conventus de Mérida était occupée par les Vettones, population souvent qualifiée de celtique dans les ouvrages généraux ${ }^{8}$. Leur territoire n'a jamais pu être

6 Tranoy, Galice, pp. 264-361; J. EnCARnaCÃo, Divindades indígenas sob o domínio romano em Portugal, Lisbonne, 1975 (= EnCARnaCÃo, Divindades); M. SAlinas de Frías, La religión de los Celtíberos (I), SHHA, II-III, 1, 1984-1985, pp. 81-101; id., La religión indígena de la Hispania central y la conquista romana, $S Z$ (Historica) VI, 1985, pp. 307-329; T. MAÑANES PÉREZ, Vacceos, dans J. M. SolanA SÁINZ (éd.), Las entidades étnicas de la Meseta norte de Hispania en época prerromana (Anejos de Hispania Antiqua), Valladolid, 1991, pp. 252-254.

7 J.J. SAyas et R. López, Vettones, dans J.M. Solana (éd.), op. cit., p. 105 (= SAYAS et LóPEz, Vettones).

8 A. Schulten et R. Grosse, Vettones, dans RE, VIII, 2, 1914, col. 1874-1875; J. Maluquer de Motes, Los pueblos de España céltica, dans R. Menéndez PidAL (dir.), Historia de España, I, 3, Madrid, 1963, pp. 24-25; J.M. BLÁZQuez, Ciclos y temas de la Historia de España: la romanización, II, Madrid, 1986, pp. 42-44; on lira également E. SÁNCHEZ MoRENO, El origen de los Vetones en la historiografía española del siglo XX. ¿Implantación o formación?, ETF, Serie II, Historia Antigua, VIII, 1995, pp. 475-499. 
délimité avec précision, faute de sources suffisantes, mais nous pensons qu'il s'étendait sur la plus grande partie des provinces de Salamanque et d'Ávila, la moitié orientale de celle de Cáceres, avec des appendices sur les provinces de Zamora, Tolède et Badajoz ainsi que sur une frange orientale du Portugal ${ }^{9}$. L'identité culturelle de cette population est de même longtemps restée dans la pénombre notamment en raison des lacunes des sources et du caractère peu homogène du peuplement de ces terres de Castille-León et d'Estrémadure. On en sait heureusement beaucoup plus depuis ces toutes dernières années grâce à un intérêt tout récent des chercheurs pour cette population ${ }^{10}$. Sans entrer dans le détail, on sait que le pays vetton était une terre de castros, sites fortifiés, et surtout de verracos, imposantes sculptures zoomorphes dont la chronologie et les fonctions sont encore source de nombreuses interrogations. Peuple éleveur fortement marqué par le nomadisme, sans doute la principale raison du caractère incertain de ses limites territoriales, les Vettons, malgré leurs productions céramiques et métallurgiques, apparaissent, à la lumière de l'archéologie (les sources littéraires étant quasiment muettes à ce sujet) comme un peuple connaissant un faible développement socio-économique et subissant fortement les influences des peuples voisins (Astures, Lusitaniens...), y compris au moment des guerres de conquêtes (IIème siècle av. J.C.).

Une fois la pacification achevée, les Romains ont procédé à l'intégration administrative de ce territoire inclus dans la province de Lusitanie, et sont entrés en contact avec un panthéon indigène qui ne nous apparaît que grâce à l'usage, introduit par les Romains dès le Ilème siècle av. J.C., de l'épigraphie.

9 Ch. Bonnaud, Vettonia Antiqua. Peuplement et société dans l'ouest de la Meseta, thèse inédite, vol I, Poitiers, 1999, pp. 19-41; idem, Vettonia Antiqua: les limites ethniques et administratives d'un peuple de l'ouest de la Meseta dans l'Antiquité, SHHA (article sous presse).

10 J.R. Álvarez-SAnchís, Los Vettones, Madrid, 1999; E. SÁnchez Moreno, Vetones: historia y arqueología de un pueblo prerromano, Madrid, 2000; M. SALINAS DE FRÍAS, Los Vettones. Indigenismo y romanización en el occidente de la meseta, Salamanque, 2001. 


\section{LES CULTES INDIGENES (cf. fig. 1) ${ }^{11}$}

\section{A. En Vettonnie du nord: des cultes indigenes dominants}

1. Prédominance des divinités indigènes à caractère inconnu dans la province de Salamanque ${ }^{12}$

Parmi les quelque 300 inscriptions latines de cette province, on a pu transcrire seulement quelques théonymes indigènes. Mais d'autres éléments de nature archéologique laissent à penser que les cultes indigènes y étaient nombreux à l'époque des Romains.

- ACPULSOIUS?: Acpulso/io?/Ontius/Primi f. En/tus/pos(uit) $)^{13}$

Cet autel aurait été retrouvé à Hornacino (Lagunilla, sud-ouest de Béjar), près des ruines d'un important castro. Malgré l'opinion de V. Soria Sánchez, il semble bien que nous soyions en présence d'un théonyme suivi d'un nom indigène ${ }^{14}$. Ce théonyme n'est apparu nulle

11 J. M. BlázQuez, Religiones primitivas de Hispania, I, Fuentes literarias y epigráficas, Rome, 1962 (= BLÁZQuEZ, Religiones); id., Diccionario de las religiones prerromanas de Hispania, Madrid, 1975 (= BlázQueZ, Diccionario); B. GARCíA FerNÁNDEZ-AlbALAT, Las divinidades indígenas de la Hispania prerromana. En pos de una metodología, TAE, XXV, 2-4, 1985, pp. 275-283; J. M. BlÁZquEZ et M. P. GARCíA-Gelabert PÉREZ, Nuevas aportaciones a las religiones primitivas de Hispania, ETF (Serie II), I, 1988, pp. 157-183; J. M. BlÁZQUEZ, Recientes aportaciones de las religiones primitivas de Hispania. Addenda y corrigenda, dans Athlon II. Satura Grammatica in honorem Francisci R. Adrados, II, Madrid, 1987, pp. 69-87; id., Primitivas religiones II: religiones prerromanas, Madrid, 1983, (= BLÁZQUEZ, Primitivas religiones); id., Recientes aportaciones a las religiones prerromanas de Hispania, Gerión, X, 1992, pp. 193-203.

12 J. M. BLÁZQUEZ, Religión y sociedad en las inscripciones de Salamanca, dans Religio Deorum. Actas del Coloquio Internacional de Epigrafía. Culto y sociedad en Occidente (Tarragona, 1988), Sabadell, 1992 (= BLÁZQUEZ, Religión y sociedad); A. Alonso Ávila et S. CRespo Ortiz de ZÁrate, Corpus de inscripciones romanas de la provincia de Salamanca, Valladolid, 1999 (= Alonso et CRESPO, Corpus); idem, Las manifestaciones religiosas del mundo antiguo en Hispania romana: el territorio de Castilla y León, I, Las fuentes epigráficas, Valladolid, 1999 (= CRESPO et ALONSO, Las manifestaciones); J.M. SOLANA SÁINZ et L. HERNÁNDEZ, Religión y sociedad en época romana en la Meseta septentrional, Valladolid, 2000 (= SOLANA et HERNÁNDEZ, Religión y sociedad).

13 Morán, Reseña, p. 69; BlázQUEZ, Religiones, p. 205; HAE, 1305; ILER, 705; BLÁZQuez, Religión y sociedad, p. 74.

14 V. Soria SÁnchez, De arqueología cacereña, dans XI CNA (Mérida, 1968), Saragosse, 1970, p. 583. 
part ailleurs dans la péninsule et sa transcription laisse planer quelque doute. L'inscription serait à situer aux IIè-IIIè siècles ${ }^{15}$.

- CAESARICIAECUS: Caesaric/iaeco/Duatius/Bovi f(ilius) fralternum $/ v$ (otum) $s$ (olvit) $l$ (ibens) $m$ (erito) ${ }^{16}$.

L'autel provient de Martiago, près de Ciudad Rodrigo. Il daterait du Ier siècle ap. J.C. ${ }^{17}$.

- CANTUNAECUS: Silo/Corai f./Cantu/naeco/v(otum) a(nimo) l(ibens) $s$ (olvit) ${ }^{18}$.

Cette divinité est apparue sur un autel du castro d' Urueña, dans le sud-ouest de la province, tout près de Ciudad Rodigo. Selon L. Albertos, la racine - Cand serait à mettre en relation avec l'idée de briller, resplendir ${ }^{19}$. L'autel daterait des IIè-IIIè siècles ${ }^{20}$.

- CELIBORCA: Baebius/Reburrus/Celiborcae/sacrae/v(otum)

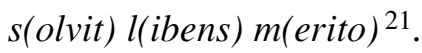

L'autel proviendrait de Villasbuenas. Selon J. M. Blázquez, il pourrait s'agir d'une nymphe.

- FAVILIUS?: Primigenius/Favilio?/v(otum) s(olvit) l(ibens) $m(\text { erito })^{22}$.

L'autel proviendrait d'Agallas. Le théonyme est incertain. Une déformation latine d'un nom de divinité indigène n'est pas à exclure.

- ILURBEDA: Ilurbeda/sacrum/Q(uintus) Maniliu[s]? Facun$d u s / v\left(\right.$ otum) s(olvit) l(ibens) a(nimo) (cf. fig. 2) ${ }^{23}$.

15 Crespo et Alonso, Las manifestaciones, p. $17 \mathrm{n} .^{\circ} 1$.

16 J. del Hoyo, Nuevos teónimos en inscripciones inéditas de la provincia de Salamanca, $M C V$, XXX (1), 1994, pp. 53-57; AE, 1994, 887; Alonso et Crespo, Corpus, p.100 n. ${ }^{\circ} 187$.

17 Crespo et Alonso, Las manifestaciones, p. 27 n. ${ }^{\circ} 14$.

18 CIL II, 861; Morán, Epigrafía, p. 56 n. ${ }^{\circ} 112$; BlázQueZ, Religiones, p. 208 (Bcantunaeco); BlázQuez, Diccionario, p. 52; BlÁZQuez, Religión y sociedad, p. 74 n. ${ }^{\circ}$; Alonso et Crespo, Corpus, pp. 30-31 n. ${ }^{\circ} 36$.

19 L. Albertos Firmat, La onomástica personal primitiva de Hispania. Tarraconense y Bética, Salamanca, 1966, p. 119.

20 Crespo et Alonso, Las manifestaciones, p. 30 n. ${ }^{\circ} 21$.

21 HaE, 1335; Maluquer, Carta, p. 139 n. ${ }^{\circ}$ 122; Blázquez, Diccionario, p. 55; Alonso et CRespo, Corpus, p. 135 n. ${ }^{\circ}$ 266; Solana et Hernández, Religión y sociedad, p. 189 et 299 n. ${ }^{\circ} 246$.

22 J. del Hoyo, art. cit., pp. 58-60; AE, 1994, 889; Hep, 5, 1995, pp. 194-195 n. ${ }^{\circ}$ 680; AlONSO et CRESPO, Corpus, pp. 168-169 n. ${ }^{\circ} 341$; idem, Las manifestaciones, pp. $43-44$ n. ${ }^{\circ} 45$.

23 BlázQuez, Diccionario, p. 109; BlázQuez, Religiones, pp. 78 ss; Mangas, 
L'autel votif de marbre blanc (0,78 m. x 0,44 m.) était inclu dans une des marches de l'église de Segoyuela de los Cornejos. Ilurbeda est également attesté ailleurs dans la péninsule, en particulier au Portugal $^{24}$. J. Mangas situe l'inscription à la fin du IIème ou début du IIIème siècle. Manilius à la place de Mineius est probable, comme le pense d'ailleurs L. A. Curchin ${ }^{25}$.

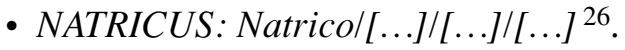

Cet autel, retrouvé à Alba de Tormes, comporte une inscription très endommagée. Le théonyme nous semble cependant assez fiable et l'étude de son étymologie (natrix), permettrait de mettre cette divinité en relation avec un culte des serpents, ces derniers ayant pu être particulièrement abondants dans les eaux du Tormes et donc l'objet d'un culte $^{27}$. D'autres interprétations ont vu le jour: il s'agirait d' une déesse de la nature et des eaux, à caractère cosmique ou guerrier, si on considère que c'est Nabia (?) qui apparaît en fait dans l'inscription: $\mathrm{Na}$ (biae) Tri(forme) Co(ronae) ${ }^{28}$. L'autel serait du Ier siècle ap.J.C ${ }^{29}$.

Nuevas inscripciones, pp. 135-136; AE, 1985, 543; Alonso et Crespo, Corpus, pp.131-132 n. ${ }^{\circ}$ 258; idem, Las manifestaciones, p. 46 n. ${ }^{\circ}$ 48; M. R. PÉrez Centeno, Los cultos indígenas en Hispania durante la dinastía Severa, dans Homenaje al prof. Montenegro. Estudios de Historia Antigua, Valladolid, 1999, p.666.

24 Encarnação, Divindades, pp. 200-203 et J. Encarnação, Divindades indígenas da Lusitânia, Conimbriga, XXVI, 1987, p. 25 (= ENCARNAÇÃo, Divindades indígenas).

25 L. A. Curchin, Epigrafic notes from the Spanish Meseta, ZPE, LVIII, 1985, p. 244 n. ${ }^{\circ} 1$.

26 J. M. Solana SÁinz et L. Sastre Varas, Nueva aportación para el estudio de la teonimía de la Hispania romana: el ara de Alba de Tormes, Durius, IV, 1976, pp. 57-60: ces deux auteurs transcrivent le nom du dédicant [Va]l(erius) Proc(ulus) [O]cta(vius), ce qui apparaît comme très aléatoire. J.M. SolAnA, Precisiones al ara de Alba de Tormes, Durius, V, 1977, p.393.

27 A. M. VÁzQUEZ HoYs, La serpiente en el mundo antiguo, BAEAA, XIV, 1981, pp. 34-39; BlázQuez, Primitivas religiones, pp. 259 ss., M. SAlinas, El toro, los peces y la serpiente. Algunas reflexiones sobre la iconografía y la religión de los Celtíberos en su contexto histórico, dans J. Mangas et J. Alvar (éd.), Homenaje a J.M. BLÁzQUEZ, II, Madrid, 1994, pp. 509-519.

28 J.L. Melena, Un ara votiva romana en El Gaitán, Cáceres, Veleia, 1, 1994, pp. 256-257; J.M. BLÁZQUEZ, Ultimas aportaciones a las religiones prerromanas de Hispania, dans Mélanges Raymond Chevalier, 2, 1, Tours, 1995, p. 69; sur Nabia, cf. Tranoy, La Galice, pp. 281-283; B. GARCÍA FERNÁNDEZ-ALBALAT, Guerra y religión en la Gallaecia y la Lusitania antiguas, La Coruña, 1990, p. 303 (= FERNÁNDEZ-ALBALAT, Guerra y religión).

29 Alonso et CRespo, Las manifestaciones, pp. 58-59 n. ${ }^{\circ} 69$. 
- TOGA: Camalus/Saelgi f./Togae/v(otum) s(olvit) l(ibens) $m(\text { erito })^{30}$.

Cette inscription provenant de Martiago, mentionne une divinité connue ailleurs dans la péninsule, avec une concentration en territoire vetton ${ }^{31}$. L'inscription daterait des IIè-IIIè siècles ${ }^{32}$.

L'archéologie vient compléter cette liste de théonymes:

- A Villa de Montemayor, également dans le sud de la province, est apparu un bloc de pierre, de forme rectangulaire, terminé par trois têtes juxtaposées (cf. fig. 3) ${ }^{33}$. Il pourrait s'agir d'une des plus importantes divinités celtiques, à en juger par le nombre de sculptures et de figures comparables retrouvées ailleurs, en particulier dans le nord de la Gaule, mais aussi en Bretagne. P. Lambrechts a cru pouvoir identifier ce dieu à trois têtes (en fait une seule tête comprenant trois visages) au Mercure romain ${ }^{34}$. On a en effet mis au jour dans plusieurs sites gaulois un dieu tricéphale avec les attributs de Mercure (caducée, sandales ailées). César insiste sur l'importance du culte du Mercure gaulois, mais sans mentionner le nom de ce dieu (Esus? Teutatès?) ${ }^{35}$. Cependant, aucun document de la péninsule ibérique ne tend à assimiler Mercure à un dieu indigène tricéphale. Aucun élément de datation n'est envisageable pour la sculpture de Villa de Montemayor.

- Non loin de là, à Candelario, une sculpture de tête à deux faces opposées, et ornée de petites cornes a été retrouvée et nous met en présence d'une nouvelle divinité dont le nom et la nature nous sont inconnus ${ }^{36}$. Cette figure pourrait représenter l'équivalent indigène du Janus bifrons ${ }^{37}$.

30 AE, 1955, 235; M. L. Albertos Firmat, Nuevas divinidades de la Antigua Hispania, Zephyrus, III, 1952, p. 62; BLÁzQUEZ, Religión y sociedad, p. 75 n. ${ }^{\circ}$ 10; Solana y Hernández, Religión y sociedad, p. 294 n. ${ }^{\circ}$ 31; Alonso et Crespo, Corpus, p. 100 n. ${ }^{\circ} 188$.

31 BlázQuez, Diccionario, p. 173; BlázQuez, Religiones, p. 127, fig. 34; J. Mangas, Religiones indígenas en Hispania, dans J. M. BLÁzQueZ et alii, Historia de España Antigua, II, Hispania Romana, Madrid, 1978, p. 600.

32 Alonso et Crespo, Las manifestaciones, pp. 70-71 n. ${ }^{\circ} 90$.

33 J. M. BlézQuez, Dios tricéfalo de la Villa de Montemayor (Salamanca), AEA, LVIII, 1987, pp. 125-128.

34 P. LAmBrechts, Contribution à l'étude des divinités celtiques, Bruxelles, 1942, p. 137.

35 CAESAR, Bellum gallicum, VI, 17-18.

36 J. Muñoz García, El Jano de Candelario, Zephyrus, IV, 1953, pp. 69-73.

37 Sur les caractères de cette divinité romaine, cf. H. de la VILLE DE MiRMONT, 
2. Une influence du panthéon lusitanien en Vettonnie portugaise $^{38}$

Plus à l'ouest, au Portugal, les divinités indigènes se retrouvent abondamment et témoignent de la proximité du pays lusitanien. Les théonymes recensés dans cette partie extrême-occidentale de la Vettonnie sont les suivants:

- AELVA?: Aelva?/Bere/c(enses?) C(astellani?) p(osuerunt) ${ }^{39}$.

Provenant probablement d'un site archéologique situé à $3 \mathrm{~km}$ de Famalicão, à Barrelas, dans la serra da Estrela, cette inscription nous livre un théonyme qu'il faut peut-être relier à Alva, théonyme connu à Mouriscas (Abrantes). Les dédicants pourraient être, selon F. Curado, une communauté montagnarde (Castellum Berecum).

- AETIUS: Ae/tio/Cis/ia l(ibens) a(nimo)/s(olvit ${ }^{40}$.

Selon J. L. I. Vaz, Aetius serait une divinité indigène seulement vénérée par une communauté ethnique restreinte car on n'en trouve nulle autre trace ailleurs.

- ARENTIA/ARANTIA: Arenti/ae Equo/tullaicen/si Nicer/Arconis (filius) $/[\ldots] /[\ldots]^{41}$.

ARANTIUS [R]ufus P/eicanilf. Arant/[i]a(e) Ocelal[e]ca(e) et A/[r]antio/[O]celaeco/[...] p(osuit)? ${ }^{42}$.

Mythologie élémentaire des Grecs et des Romains, Paris, 1910, pp. 77-79. L. AdAMS Holland, Janus and the bridge, Rome, 1961.

38 Encarnação, Divindades; EnCARnaÇão, Divindades indígenas, pp. 7-37; on n'hésitera pas non plus à relire J. LEITE DE VASCONCELOS, Religióes da Lusitânia, I-III, Lisbonne, 1897-1913 ainsi que P. BÁRCIA, As "Religióes da Lusitânia" de J. Leite de Vasconcelos: contribuição para a seu estudo. Alguns comentários e índices gerais, Lisbonne, 1982; J. de AlARCÃO, Divindades da Beira: ensaio da geografia religiosa, dans Arqueologia Hoje, I, 1990, pp. 146-169; J. M. GARCIA, Religiões Antigas de Portugal. Aditamentos às Religiões de Lusitânia. Fontes epigráficas, Lisbonne, 1991.

$39 A E, 1988,699$ («interprétation désespérée») F. CURADO, Ara a Aelva, de Famalicão (Guarda), FE, 22, 1987, n. ${ }^{\circ}$ 98; ENCARNAÇÃO, Divindades indígenas, p. 15.

$40 A E, 1977$, 354; J. L. I. VAZ, Inscrições Romanas do Museu do Fundão, Conimbriga, XVI, 1977, pp. 6-7 n. ${ }^{\circ} 1$ (= VAZ, Inscrições).

$41 A E, 1984,479 ;$ F. CURAdo, Monumento votivo a Arentia, de Sabugal, FE, 7 , 1984, n. $^{\circ} 27$.

42 Alarcão, Roman Portugal, II, 1, n. ${ }^{\circ}$ 4/368; J. Mendes de Almeida e F. BANDEIRA FERrEIRA, Varia Epigraphica, $R G$, LXXIX, 1969, pp. 257-260. 
Mise au jour à Sabugal, la première inscription, qui pourrait dater de la première moitié du IIème s. ap.J.C., nous met en présence d'une divinité relativement bien connue en pays lusitanien, bien que n'étant pas accompagnée ici de son équivalent masculin ${ }^{43}$. L'épithète demeure obscure en dépit des tentatives d'interprétation ${ }^{44}$. Le deuxième document provient de Ferro, au sud-est de Covilhã, et les deux divinités devaient être adorées dans la zone d'Ocelum ${ }^{45}$.

- BANDIS ARBARIAICUS: Amminus/Andaitiae f./Bandi Arbal Iriaico vo/tum l(ibens) $m$ (erito) $s$ (olvit) ${ }^{46}$.

- BANDEIS BRIALEACUS: Bandei/Brialeac/ui Severu/s Abruni f./v(otum) $s(\text { olvit })^{47}$.

- BANDIS ISIBRAIA: Bandi/Isibraie/Cilius/Camali/f(ilius) $v$ (otum) $s$ (olvit) ${ }^{48}$.

[M]ater[nus] M[ae]/lon(is)/f. Ba[n]di Is(i)b[ra(ie)] /v(otum) s(olvit) l(ibens) [m(erito) $]^{49}$.

- BANDIS VORTEAECEUS?: Reburrus/Tangini (filius)/Bandi Vo/rteaeceo $v$ (otum) $s$ (olvit ${ }^{50}$.

- VORTIAECIUS: Fla(vius) Turi/us Felix/Vortiaecii?/v(otum) $s(\text { olvit })^{51}$.

Ces inscriptions nous mettent en présence d'une divinité bien connue de l'ouest ibérique puisqu'on a recensé environ une trentaine de

43 Encarnação, Divindades, pp. 97-108; J. DE Alarcão, Novas perspectivas sobre os Lusitanos (e outros mundos), Revista Portuguesa de Arqueologia, IV, 2, 2001, p. 303 (notamment la carte) (= ALARCÃo, Novas perspectivas).

44 L'épithète pourrait être géographique, en relation avec le site de Sabugal.

45 Sur cette divinité en relation avec le toponyme Ocelum, $c f$. M. L. AlBERTOS FIRMAT, A propósito de algunas divinidades lusitanas, dans Symbolae Ludovico Mitxelena Septuagenario Oblatae, Vitoria, 1985, pp. 470-474 (= AlberTos, Divinidades lusitanas).

46 CIL II, 454 (Capinha); ILER, 753; EnCARnAÇÃo, Divindades, pp. 129-131; ENCARNAÇÃo, Divindades indígenas, p. 19.

47 AE, 1967, 135; ENCARNAÇÃo, Divindades, pp. 125-126 (Orjais); ENCARNAÇÃO, Divindades indígenas, p. 19.

48 \& 49 AE, 1967, 133 et 134; EnCARnAÇÃo, Divindades, pp. 131-132 (Bemposta); EnCARnaÇão, Divindades indígenas, p. 19; M. LeItão et L. BARATA, Inscrições romanas de Bemposta, TAE, XXIII, 1980, pp. 627-631.

50 VAZ, Inscrições, pp. 8-9 n. ${ }^{\circ}$ III (Salgueiro); EnCARnAÇÃo, Divindades, pp. 137-138.

$51 A E, 1985,531$; F. Curado, Ara a Vortiaecius, de Penamacor, FE, 13, 1985 , n. ${ }^{\circ} 57$. 
théonymes formés sur la racine Band ${ }^{52}$. Bandis/Bandeis pourrait être une divinité tutélaire ${ }^{53}$. On notera enfin l'absence de ce théonyme devant Vortiaecii?, lequel apparaît plus comme un théonyme à part entière qu'une épithète sur l'autel d'Arrochela (Penamacor).

- CORVA: Corvae Pat(ernus?)/ex v(oto) patris/a(ram) p(osuit $)^{54}$.

Près du sanctuaire chrétien de Nossa Senhora da Marofa (Castelo Rodrigo), on a découvert cet autel dédié à une déesse inconnue jusqu'ici alors, sauf peut-être dans le nord-ouest ${ }^{55}$.

- LAEPUS: Laepo/Firmu[s]/Sabinae/l(ibertus) v(otum) l(ibens) $s(\text { olvit })^{56}$.

Tanginus L(ucii) Bouti(i) (filius)/Laepo a(nimo)/l(ibens) v(otum) s(olvit) ${ }^{57}$.

Laepo/v(otum) s(olvit)/Bassu/s Virialti f(ilius) ${ }^{58}$.

52 J. M. BlázQuez, Religiones, p. 478; BláZQuez, Diccionario, p. 46 (carte); Encarnação, Divindades, pp. 119 ss.; F. B. FerreIra et J. M. de AlmeIdA, Uma Arula a Banduaetobricus, Conimbriga, XV, 1976, pp. 139-146; TrAnOY, Galice, pp. 279-280; J. SAlas MARTín et alii, Bandia Apolosegus, una divinidad con culto local en la zona de Brozas, Cáceres (Nuevas aportaciones epigráficas), HAnt, XIII, 1986-1989, pp. 7-20; FernándeZ-Albalat, Guerra y religión, pp. 124 ss.; Alarcão, Novas perspectivas, fig. 6 et p. 305; AE, 1977, 380 recense une inscription à [...Bandi] Vor[te]aecio à São Martinho, au sud-est de Castelo Branco; $c f$. également F. SANDE LEMos et J. ENCARnAÇão, Ara votiva a Bandu Vordeaeceo, FE, 40, 1992, n. ${ }^{\circ} 179$ (à Quinta da Ribeira, district de Bragance).

53 ENCARNAÇÃo, Banda, uma importante divindade indígena, Conimbriga, XII, 1973, pp. 199-214; J. M. GARCIA, Contributo para a compreensão das divindades do “grupo Band”. Uma nova ara, Conimbriga, XV, 1976, pp. 147-150; BLÁZQUEZ, Religiones, pp. 55-57; M. SALINAS, La religión indígena del oeste de la Meseta: los Vettones, SZ (Historia), III, 1982, p. 328; R. PEDRERO, Los epítetos del teónimo occidental Bandue/i, dans Religión, lengua y cultura prerromanas de Hispania. VIII Coloquio sobre lenguas y culturas prerromanas de la Península Ibérica (Salamanca, 1999), Salamanque, 2001.

54 AlarCão, Roman Portugal, II, 1, n. ${ }^{\circ}$ 4/95; A. CABral, Ara votiva do castro da Marofa, Beira Alta, XXVIII, 1969, pp. 3-11; F. CurAdo, Epigrafia das Beiras (Notas e correcções), Beira Alta, XLIV, (4), 1985, pp. 650-651.

55 CIL II, 5594; Tranoy, Galice, p. 273 (CORV [...] sur un rocher de Briteiros).

56 Alarcão, Roman Portugal, II, 1, n. ${ }^{\circ} 4-304 ;$ F. CURAdo, Aras a Laepus procedentes de Pousafoles, Sabugal, FE, 7, 1984, pp. 8-12; HEp, 2, 1990, 794.

$57 A E, 1984,480$.

$58 A E, 1984,481$. 
Laepus est attestée sur trois inscriptions parmi 14 autels anépigraphes (sanctuaire ou atelier?) de Quinta de São Domingos (Pousafoles do Bispo). On retrouve également la trace de ce dieu sur l'inscription lusitanienne de Cabeço das Fráguas, un peu plus à l'ouest ${ }^{59}$. Selon K.T. Witczak, Laebo/Laepo serait l'équivalent lusitanien du datif pluriel laribus ${ }^{60}$.

- LANEANA? : Laneane?/Tang(inus)? f(ecit)? ${ }^{61}$.

Ce possible théonyme (ou épithète de théonyme?) était gravé sur un affleurement rocheux près d'Aldeia da Ponte (Sabugal). On le retrouverait dans la province de Cáceres, à Fuentes de Higuera ${ }^{62}$.

- M.B.S.: M.B.S(acrum)/Iunia/Firmina/a(nimo) l(ibens) v(otum) $s(\text { olvit })^{63}$.

Cette abréviation a pu être localisée sur une inscription de Paranhos da Beira (Guarda), près de l'église. Le nom de la divinité n'a pu être identifié.

- QUANGEIUS: Caturo/Tureiu (filius) deo/[.]uangeio/v(otum) s(olvit) ${ }^{64}$.

Tanginus/Turani f./Quangeiu/v(otum) s(olvit) ${ }^{65}$.

$[\ldots] /\left[f(\right.$ ilius $)$ Qu]angei[o?]/a(nimo) l(ibens)/v(otum) s(olvit) ${ }^{66}$.

Outre Penamacor et Ribeira da Nave, Quangeius est également attesté à Malpartida de Plasencia (province de Cáceres), à Capinha et, hors de Vettonnie, à Nisa ${ }^{67}$.

59 A. Tovar, La inscripción de Cabeço das Fráguas y la lengua de los Lusitanos, dans Actas del III Coloquio sobre Lenguas y Culturas Paleohispánicas (Lisboa, 1980), Salamanque, 1985 , pp.227-253

60 K.T. WitcZAK, On the indo-european origin of two Lusitanian theonyms (Laebo and Reve), Emerita, LXVII, 1, 1999, pp.68-70.

$61 A E, 1988,694$ (il s'agirait d'une divinité protectrice de la fontaine voisine); F. Curado, Inscrição rupestre de Aldeia da Ponte (Sabugal), FE, 22, 1987, n. 99.

62 C. Callejo Serrano, Aportación a la epigrafia romana del Campo Norbense, BRAH, CLVII, 1965, p. 22.

$63 A E, 1986,297$; F. Curado, Ara votiva de Paranhos da Beira, FE, 17, 1986, n. ${ }^{\circ} 76$.

64 AE, 1984, 478; F. CuRAdO, Ara a Duangeius de Penamacor, FE, 7, 1984, n. 26.

$65 A E, 1988,695$; F. CuRAdo, Aras a Quangeius da Ribeira da Nave (Sabugal), FE, 22, 1987, n. ${ }^{\circ} 100$.

$66 A E, 1988,696 ;$ F. CURADO, Aras a Quangeius da Ribeira da Nave (Sabugal), $F E, 22,1987$, n. $^{\circ} 100$.

67 EnCARnaÇão, Divindades indigenas, p. 29; FE, 23, n. ${ }^{\circ} 103$; AlarCão, Novas perspectivas, p. 303 (carte) et 305 . 
- R.C.: $\quad[$ Ca]sabius/[T]urrilli $\quad$ f./v(otum) $\quad$ s(olvit) l(ibens) $m($ erito $) / R() C()^{68}$.

Datée du IIème s. ap.J.C., cette inscription a sans doute été rédigée en l'honneur d'une divinité locale à en juger par l'abréviation qui devait être composée des initiales du théonyme et de l'épithète. Il pourrait s'agir de Reva, également mentionné sur l'inscription de Cabeço das Fráguas et surtout dans le nord-ouest, d'où Santa Eufémia n'est pas très éloignée. En outre, en Galice, Reva est toujours cité en compagnie d'un qualificatif masculin ${ }^{69}$.

- REUS PARAMAECUS?: [Ru]fus T/[...] f(ilius ex) v/(oto) [de $\mid R e$ ?]o. Pal[ra]maeco?/(aram posuit?) ${ }^{70}$.

$\mathrm{Si}$ on accepte cette délicate transcription de F. Curado, nous aurions ici un témoignage d'une divinité également présente dans le nord ouest, à Lugo plus précisément, où son culte était pratiqué aux IIème et IIIème siècles ${ }^{71}$. $s($ olvit $){ }^{72}$.

- [...]AMOI?: [...]amoi?/Cami/ra Boulti f(ilia)/v(otum) l(ibens)

Le théonyme est incomplet et difficilement identifiable.

\section{Près d'Ávila: un sanctuaire à Endovellicus}

L'Avela romaine a fourni peu de traces de cultes indigènes, en dépit de la faible influence romaine dans la région ${ }^{73}$. De nombreuses divinités indigènes devaient cependant y être adorées sans avoir laissé de témoignages épigraphiques abondants, traduisant ainsi la persistance

68 AE, 1983, 475; Alarcão, Roman Portugal, II, 1, n. ${ }^{\circ} 4 / 128$; M. L. LoPES ToMÉ, Uma inscrição votiva de Santa Eufémia (Pinhel), FE, 5, 1983, n. ${ }^{\circ} 17$.

69 Blázquez, Religiones, pp. 185-187; BlázQuez, Diccionario, p. 141; TrANOY, Galice, p. 285.

70 F. Curado, Ara fragmentada de Fóios (Sabugal), FE, 27, 1988, n. ${ }^{\circ} 122$.

71 Tranoy, Galice, p. 290. L'épithète évoque un anthroponyme présent à Marialva (Meda): Paramaecus.

72 AE, 1977, 356; HEp, 3, 1991, 471; VAZ, Inscrições, pp. 10-11 n. ${ }^{\circ}$ IV (Capinha).

${ }^{73}$ R.C. KNAPP, Latin Inscriptions from Central Spain (Classical Studies, XXXIV), Berkeley - Los Angeles - Oxford, 1992, p. 405 (= KNAPP, Latin Inscriptions); M. MAriné, La época romana, dans M. Mariné (coord.), Historia de Ávila, I: Prehistoria e historia antigua, Ávila, 1998, pp. 283-338. 
de pratiques religieuses préromaines non fondées sur l'écrit. Une autre explication est que le matériel épigraphique retrouvé à Ávila provient de la nécropole voisine; par conséquent, il s'agit presque totalement de stèles funéraires par la suite incorporées à la muraille médiévale ${ }^{74}$. On connait néanmoins l'existence de cultes voués à quelques rares dieux: $T o(. .$.$) et Maiduana.$

- Deo To(goti?) v/otum et/ara(m)/Val(erius) Mater/nus [... $]^{75}$. L'autel est perdu et la formule votum et aram est peu commune. En outre, E. Rodríguez Almeida suggère que cette inscription serait en fait la pièce incorporée dans le secteur ouest de la muraille, à ras du sol, dans la troisième tour à gauche de la Puerta de San Segundo ${ }^{76}$. Or, ce document, bien que son inscription soit aujourd'hui illisible, présente des motifs (lune avec «cornes» dirigées vers le haut) qui inciteraient à voir là une stèle funéraire et non un autel votif. Cependant, l'hypothèse d'un seul et même document est des plus fragile et ce, d'autant plus qu'à Talavera de la Reina, Togotis (ou Togos) est formellement attesté, ainsi que dans l'inscription celtibère de Botorrita (Sarragosse) ${ }^{77}$. R. C. Knapp, pour sa part, bien que n'ayant pu retrouver le document à l'endroit précisé par $\mathrm{F}$. Fita à la fin du siècle dernier, n'émet aucun doute sur son authenticité ${ }^{78}$. On sait peu de choses sur ce théonyme qui pourrait être assimilé au dieu lusitanien Tongo, "dieu de la source par laquelle on jure" 79.

\section{Maiduanae/Animpis/Minervae ${ }^{80}$}

Une divinité indigène (Maiduana) associée à des dieux romains

74 E. Rodríguez AlmeIdA, Ávila romana, p. 47, 87-95; KNAPP, Latin Inscriptions, p. 3.

75 CIL II, 5861; RodríGUEZ AlmeIdA, Ávila romana, p. 148 n. ${ }^{\circ} 63$; KNAPP, Latin Inscriptions, pp. 11-12 n. ${ }^{\circ} 3$.

76 E. RodríGUEz AlmeIdA, Ávila romana, pp. 130-131 n. ${ }^{\circ} 44$.

77 CIL II, 893; ILER, 934-935; BLÁZQUEZ, Religiones, pp. 126-127; BLÁZQUEZ, Diccionario, p. 173.

78 F. FITA, Noticias: Ávila, BRAH, XIII, 1888, pp. 334-335; J. DíEz AsEnsio, Teonimía indígena en las tierras meridionales del Duero medio, HAnt, XIX, 1995, p. 13, précise que le radical togi signifie "aimable" "agréable" en celte; d'autres auteurs pensent qu'il s'agit d'une déformation du celte tong qui signifie "jurer".

79 Tranoy, Galice, pp. 283-285.

80 Rodríguez Almeida, Ávila romana, p. 175 n. ${ }^{\circ}$ 98; J. DíEz Asensio, art. cit., p. 11 . 
est décelable dans cette inscription atypique au sein de la production avilaise ${ }^{81}$.

Velicus (ou Vaelicus) adaptation locale d'Endovellicus, est particulièrement bien représenté dans cette région nord-orientale de la Vettonnie. En effet, il existait un sanctuaire consacré à ce dieu à Postoloboso, non loin du castro préromain de Candeleda ${ }^{82}$, à la limite des actuelles provinces d'Ávila, de Tolède et de Cáceres. La géographie du lieu a été décrite par F. Fernández Gomez: à la confluence de deux cours d'eau qui se jettent dans le Tiétar, au pied d'une hauteur dénommée Cabeza de San Juan ${ }^{83}$. Le lieu semble avoir été depuis longtemps imprégné d'un caractère sacré et on y trouve aujourd'hui un ermitage.

Le matériel épigraphique est essentiellement constitué par un important lot de 19 autels, dont 9 ont été utilisés dans les murs de l'actuel ermitage ${ }^{84}$. Les autels lisibles ont livré les dédicaces suivantes (cf. fig. 4):

- Eburein/ius Curun/dif. Caraleciq(um) Vaeli/co v(otum) s(olvit) $m$ (erito) l(ibens $)^{85}$.

- Vae[li]co/sacrum/Atta Bouti/Mene[t]qui/qum f. v(otum) s(olvit)/l(ibens) $m$ (erito) ${ }^{86}$.

- Culantiu[s]/Pintolanc(um)/Velico aram/e(x) v(oto) l(ibens) a(nimo) $p(\text { osuit })^{87}$.

- Deo Velico/Marcia H/elene po(suit)/[l(ibens)] a(nimo) v(otum) $[s(\text { olvit })]^{88}$.

81 La pierre est poreuse et âpre; les lettres sont fines et encadrées par des lignes servant de repères; il faut suspecter une autre origine qu'Ávila. La provenance exacte de cette inscription est inconnue.

82 F. FERnÁndez GoMez, Excavaciones arqueológicas en el Raso de Candeleda (Ávila), I et II, 1986, Ávila (= FERNÁNDEZ GoMEZ, Excavaciones) est l'étude la plus complète sur le site.

83 FERNÁNDEZ GómEZ, Excavaciones, pp. 879-880.

84 F. FERnÁNDEZ GómEZ, El santuario de Postoloboso (Candeleda, Ávila), NAH, II, 1974, pp. 169-270 (= FERNÁNDEZ GóMEZ, El santuario).

85 KnAPP, Latin Inscriptions, pp. 97-98 n. ${ }^{\circ}$ 109; FernÁndez GómEZ, Excavaciones, pp. 882-884 n. ${ }^{\circ} 1$.

86 AE, 1976, 343, FERNÁNDEZ GÓMEZ, El santuario, pp. 212-215 n. 2; FERNÁNDEZ GómEZ, Excavaciones, p. 885 n. ${ }^{2}$ 2; KNAPP, Latin Inscriptions, p. 87 n. ${ }^{\circ} 97$ : a(nimo) pour le dernier mot.

87 AE, 1976, 344; FERNÁNDEZ GómEZ, El santuario, pp.215-216 n. ํ 3; FERNÁNDEZ GómEZ, Excavaciones, p. 886 n. ${ }^{\circ}$ 3, KNAPP, Latin Inscriptions, pp. 87-88 n. 98. 88 AE, 1976, 345; FERNÁNDEZ GóMEZ, El santuario, pp. 217-218 n. ${ }^{\circ}$; 
- Deo Veli/co s[a]crum/Mirtuole[...]o/v(otum) s(olvit $)^{89}$.

- Velico/Sentia/[...] $\operatorname{aram} /[\ldots]^{90}$.

- $D($ eo $) V($ elico $) / l\left(\right.$ ibens) $v$ (otum) s(olvit) $[\ldots]^{91}$.

- $D($ eo $) V($ elico $) / l\left(\right.$ ibens) v(otum) s(olvit)/ex v/oto ${ }^{92}$.

- [...]/cius Celer/l(ibens) a (nimo) v(otum) s(olvit $)^{93}$.

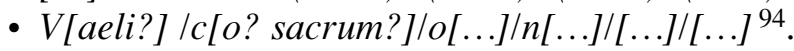

- Anna d(eo)/Velicolex [...]/voto 95 .

- $D($ eo $) V($ elico $) s($ acrum $) /[\ldots] /[\ldots] /++A+{ }^{96}$.

Cet ensemble épigraphique est unique dans toute la Vettonnie. Il prouve en effet qu'il existait, aux IIème et IIIème siècles ap. J.C. un important sanctuaire dans la localité de Postoloboso. Outre les traces épigraphiques, le site a également livré des témoignages archéologiques d'intérêt:

- Un $e x$-voto de bronze ibérique représentant une statuette masculine d'environ $11 \mathrm{~cm}$ de hauteur (cf. fig. 5). Le personnage représenté a une des deux mains recouvrant les parties sexuelles, la tête légèrement penchée vers l'arrière, les yeux fermés et en forme d'amande, la tête couverte d'une coiffe, la bouche fermée mais marquée d'un rictus

FERNÁNDEZ GómEZ, Excavaciones, p. 886 n. ${ }^{\circ}$ 4, KNAPP, Latin Inscriptions, pp. 87-88 n. ${ }^{\circ} 99$.

89 AE, 1976, 346; FERnÁNDEZ Gómez, El santuario, pp. 217-218 n. ${ }^{\circ}$ 5; FERNÁNDEZ GómEZ, Excavaciones, p. 886 n. ${ }^{\circ}$ 5, KNAPP, Latin Inscriptions, pp. 87-88 n. ${ }^{\circ} 100$.

90 AE, 1976, 347; Fernández Gómez, El santuario, p. 220 n. ${ }^{\circ} 6$ (Fentia); FeRNÁNDEZ GómEZ, Excavaciones, pp. 887-888 n. ${ }^{\circ} 6$ (Fentia); KNAPP, Latin Inscriptions, pp. $90-91 \mathrm{n} .^{\circ} 101$.

91 Fernández Gómez, El santuario, p. 221-222 n. ํ 7; FERnández Gómez, Excavaciones, p. 888 n. ${ }^{\circ}$ 7; KNAPP, Latin Inscriptions, pp. 91-92 n. ${ }^{\circ} 103$.

$92 A E$, 1976, 348; FERNÁNDEZ GómEZ, El santuario, p. 225 n. ${ }^{\circ}$ 13; FERnÁNDEZ GómEZ, Excavaciones, pp. 889 n. ${ }^{\circ} 13$; KNAPP, Latin Inscriptions, p. 91 n. ${ }^{\circ} 102$.

93 AE, 1976, 349; FERnÁNDEZ GómEZ, El santuario, pp. 225-226 n. 14 (C+Vsceles); FERnÁNDEZ GóMEZ, Excavaciones, pp. 889-890 n. ${ }^{\circ} 14$; KNAPP, Latin Inscriptions, pp. 93-94 n. ${ }^{\circ} 106$.

94 FERnÁndez Gómez, El santuario, pp. 226-227 n. ํ 15; FERnÁNDEZ GómEZ, Excavaciones, p. 890 n. ${ }^{\circ}$ 15; KNAPP, Latin Inscriptions, pp. 92-93 n. ${ }^{\circ} 104$.

95 FERnÁndez GómEZ, El santuario, pp. 222-223 n. ${ }^{\circ}$ 8; FERnÁndEZ GómEZ, Excavaciones, p. 888 n. ${ }^{\circ}$ 8; KNAPP, Latin Inscriptions, p. 86 n. ${ }^{\circ} 96$.

96 Fernández Gómez, El santuario, p. 224 n. ${ }^{\circ}$ 9; Fernández Gómez, Excavaciones, pp. 888-889 n. ${ }^{\circ}$; KNAPP, Latin Inscriptions, p. 93 n. ${ }^{\circ} 105$. 
sévère. Le caractère peu travaillé de la figurine empêche de voir si le personnage est représenté habillé ou non, mais le geste de la sculpture suggère qu'il est nu. Ce personnage est-il en position rituelle, son geste ayant été mis en exergue, au détriment de l'aspect esthétique de l'objet? L'attitude surprenante de la statuette est difficile à interpréter ${ }^{97}$. Cette dernière est, semble-t-il, unique dans tout l'art de la péninsule ${ }^{98}$. Une hypothèse serait de mettre le geste de la figurine en relation avec le thème de la fécondité ou de la maladie ${ }^{99}$. Quant à l'individu représenté, il pourrait s'agir, si l'on se réfère à G. Nicolini, d'un prêtre ${ }^{100}$. La fonction d'ex-voto de l'objet est probable mais elle semble alors peu compatible avec l'hypothèse d'une représentation de prêtre ${ }^{101}$. La datation de la statuette demeure très incertaine. Le lieu précis de la trouvaille n'est pas accessible et on peut envisager un usage de cet objet sur une très longue période, jusqu'à la christianisation du lieu.

- Des tessons de céramique sigillée des IIème-IIIème siècles mais aussi plus tardifs (Vème siècle) ${ }^{102}$. Ils ont pu appartenir à des vases à usage rituel et témoignent d'une occupation du lieu au moins jusqu'au Bas-Empire.

Finalement, il nous faut insister sur le caractère éminemment sacré du site, malgré l'absence de structures (autres que l'ermitage chrétien) tels qu'autel de sacrifice, temple, ou simple enceinte sacrée. Il est possible que même sous la domination romaine (la plupart des autels votifs ont été datés des IIème et IIIème s.), les adeptes de ce culte soient restés fidèles au caractère naturaliste de leurs croyances ${ }^{103}$. En outre, il ne fait guère de doute que la divinité adorée en ce lieu était une

97 FERnÁndez GómeZ, Excavaciones, pp. 891-894.

98 Sur ces postures, $c f$. G. NiCOLINI, Gestes et attitudes cultuels des figurines de bronze ibériques, $M C V$, IV, 1968, pp. 27-50 et fig.13 (la plus proche de notre figurine); BlÁzQuez, Diccionario, p. 160.

99 G. NicOLINI, art. cit., p. 42.

100 G. Nicolini, Les bronzes figurés des sanctuaires ibériques, Paris, 1969, p. 138 .

101 Il est plus logique de penser qu'un ex voto représente un fidèle (ou plus précisément la représentation de la partie de son corps guéri par le dieu) et non un prêtre; en outre BLÁZQUEZ, Diccionario, p. 157, rappelle que la posture habituelle du prêtre est de laisser tomber les mains le long du corps, les paumes ouvertes.

102 FERnández Gómez, Excavaciones, pp. 895-897.

103 FERnÁNDEZ Gómez, Excavaciones, p. 965. 
variante vettonne du dieu lusitanien Endovellicus et que cette divinité était vénérée (et consultée?) à cet endroit par une population qui apparaît comme socialement hétérogène ${ }^{104}$.

Mais comment expliquer la présence de cet ex-voto tout à fait particulier dans un site consacré à un dieu infernal ${ }^{105}$ ? On peut envisager plusieurs réponses à cette question:

- Le caractère guérisseur d'Endovellicus peut expliquer la posture de la figurine ${ }^{106}$.

- Endovellicus étant le dieu de la vie d'outre-tombe, celui qui confère à ses fidèles la vie éternelle; l'ex-voto pourrait être lié au désir d'un fidèle de conserver une certaine forme de fécondité dans l'au-delà ${ }^{107}$.

- Il est possible que cette statuette soit sans lien véritable avec le culte d'Endovellicus, ne serait-ce que pour de simples raisons chronologiques, d'autres formes de dévotion ayant pu précéder l'instauration d'un sanctuaire dédié à Endovellicus en ce lieu.

- Hors de son contexte d'origine (ibérique?), cette figurine n'a peut-être tout simplement aucune valeur religieuse (jouet?) et ne serait qu'un témoignage de contacts entre le sud péninsulaire et le monde vetton.

\section{B. En Estremadure vettonne: une bonne resistance des cultes in- digenes}

\section{En Haute-Estrémadure}

La région la plus septentrionale de la province de Cáceres, dominée par la sierra de Gata ainsi que son versant méridional (bassins de

104 BlázQuez, Diccionario, p. 95; J. EnCARnAÇÃo, Inscrições Romanas do Conventus Pacensis, Coimbre, 1984, pp. 800-805 (sur São Miguel da Mota). On consultera d'autres autels consacrés à ce dieu dans J. ENCARNAÇão, Textos fragmentados em honra de Endovélico, FE, 3, 1982, n. ${ }^{\circ} 10$.

105 ENCARNAÇÃo, Divindades, pp. 181-185, fait le point sur ce que nous savons de ce dieu.

106 S. LAmBrino, Le dieu lusitanien Endovellicus, BEP, XV, 1950, pp. 116-117, critique la fonction purement médicale du dieu et assimile ce dernier au Sucellus gaulois; sur l'aspect guérisseur d'Endovellicus, $c f$. G. NiCOLINI, art.cit., p. 43.

107 S. LAmbrino, Les cultes indigènes en Espagne sous Trajan et Hadrien, dans Colloque sur les Empereurs Romains d'Espagne (Madrid, 1964), Paris, 1965, p. 230 (= LAMBrino, Les cultes indigènes); G. Nicolini, art. cit., p. 42. 
l'Alagón et du Tiétar), au nord du Tage, se révèlent être d'excellents conservatoires des cultes indigènes ${ }^{108}$. C'est particulièrement vrai de la zone la plus occidentale de la sierra de Gata où se situe son point culminant: le Mont Jálama (1492 m).

- ARABOCOROBA EICOBUS? TALUSICUS?: Arabo/corobelEicobo?/Talusico?/[...]/m.t.d./d. m./[...]m[...]? ${ }^{109}$.

A Arroyomolinos de la Vera, un autel a livré un ou des théonymes inconnus jusqu'alors; il est tout à fait possible que l'inscription évoque plusieurs divinités ${ }^{110}$.

- ASITRITA?: L. Covelus? Boluti f. A/sitritaelv(otum) s(olvit) l(ibens) $m$ (erito) ${ }^{111}$.

[...]/[...] Asi/(tritae?) Raphi/ciogisae?/v(otum) s(olvit) l(ibens) a(nimo $)^{112}$.

Cette divinité est attestée à Navaconcejo et, peut-être, à Malpartida de Plasencia. Asitrita est inconnue, semble-t-il, dans le reste de la péninsule. S'agirait-il d'une adaptation locale de la déesse Astarté?

- BANDIS? VORT[IA]CIUS?: [...]/[...]/Band[...] Vort[...]ciol $/$ Ca[t]/uenus [...]/[...] v(otum) s(olvit)/l(ibens) m(erito ${ }^{113}$.

Si l'épithète demeure très incertaine - CIL II, 855 lit Bandis Vorp [...]icius, nous retrouvons dans la province de Cáceres, à Malpartida de Plasencia, une divinité tutélaire particulièrement vénérée en pays lusitanien: Bandis.

108 M. García De Figuerola, Arqueología romana y altomedieval de la sierra de Gata (El valle del Valverde, provincia de Cáceres), Cáceres, 1999, pp.123-132 et pp. 157-162.

109 M. Beltrán LloRis, Aportaciones a la epigrafía y arqueología romana de Cáceres, Caesaraugusta, XXXIX-XL, 1975-76, pp. 76-77 n. ${ }^{\circ} 52$ et fig. 45 (= BELTRÁN, Aportaciones); pour les dernières lignes, cet auteur propose sans conviction m(emoriae?) t(itulum?) $d$ (onavit), d(ivino) $m$ (andatu), proposition très peu crédible; on notera que Aracoaranius est attesté sur un autel lusitanien: CIL II, 722.

110 J.C. Olivares Pedreño, El panteón religioso indígena del área extremeña, Hant, XXIII, 1999, p.109, transcrit Arabocorobe Eicobo Talusico et y voit un théonyme et deux épithètes (= OLIVAREs, El panteón). p. 109.

111 ILER, 731; Blázquez, Religiones, pp. 206-207; Olivares, El panteón,

112 Blázquez, Diccionario, p. 30; Sayas et López, Vettones, p. 112 n. 62; ILER, 911.

113 OLIVARES, El panteón, pp. 99-100. 
- QUANGEIUS?: Mearus/Meidueni f./v(otum) s(olvit) D(eo)/ /Quangeio?/l(ibens) a (nimo) ${ }^{114}$.

Cet autel votif provenant également de Malpartida de Plasencia fait sans doute référence à une divinité bien connue au Portugal.

- PALANTICUS: Palentico/Scanus Angeliti f. v(otum) s(olvit) l(ibens)/m(erito) ${ }^{115}$.

A Perales del Puerto, cet autel, longtemps considéré comme perdu, était scellé à l'intérieur de l'église. Pourrait-il s'agir de la divinité protectrice du populus des Palantienses ${ }^{116}$ ?

- SALAMA: Fuscus Deo/Salamati/v(otum) s(olvit) l(ibens) $m$ (erito) ${ }^{117}$.

Salama/Leurius/Bouti f./v(otum) s(olvit) ${ }^{118}$.

Provenant de Villamiel et de Ceclavín, ces deux inscriptions sont dédiées à Salama, théonyme qu'il conviendrait de mettre en relation avec le Mont Jálama, au pied duquel se localise Villamiel, Ceclavín se trouvant un peu plus au sud. M. L. Albertos Firmat et J. L. Melena ont montré que la montagne et la divinité portaient le même nom dans l'Antiquité (Salama) et que le Mont Jálama était divinisé comme l'étaient de nombreux autres pics de l'ouest péninsulaire ${ }^{119}$. Il faut donc mettre fin à la tradition qui voit dans Salama un dieu à caractère aquatique, concrètement le Tormes, d'où serait dérivé le toponyme Salmantica ${ }^{120}$. Nous verrons qu'un troisième témoignage de ce théonyme est possiblement attesté à Trujillo.

114 AlarCão, Novas perspectivas, p. 305; ILER, 802 et BláZQUEZ, Diccionario, p. 90 lisaient Dovanceius.

115 A. SÁnchez PAREdes, Inscripciones latinas de la Vettonia, dans V Congreso de Estudios Extremeños (Arqueología y Arte Antiguo), Badajoz, 1976, pp. 91-94 (Scitus); J. L. MelenA, Salama, Jálama y la epigrafía latina del antiguo corregimiento, dans Symbolae L. Mitxelena Septuagenario Oblatae, Vitoria, 1985, p. 494 n. ${ }^{\circ} 14$ (= MELena, Salama); BlázqueZ, Diccionario, p. 137; Hep, 1, 1989, 177; Hep, 6, 1996, n. ${ }^{\circ} 239$.

116 Sur ce populus, cf. AlaRCÃo, Novas perspectivas, pp. 308-309.

117 Melena, Salama, p. 479 n. ${ }^{\circ} 5$.

118 Melena, Salama, pp. 481-483 n. ${ }^{\circ} 7$.

119 Albertos, Divinidades lusitanas, pp. 469-470; Melena, Salama, pp. 524-526; M. L. Albertos Firmat, El culto a los montes entre los Galaicos, Astures y Berones y algunas de las deidades más significativas, EAA, VI, 1974, pp. 147-157.

120 A. Tovar, Toponimos con - $n t$ en Hispania y el nombre de Salamanca, dans Actas del V Congreso Internacional de Ciencias Onomásticas (Salamanca, 1955), II, Salamanque, 1958, pp. 114 ss. 
- SELAES DUILLAS?: [...]/votum fecit libe(nter) Selais Duil(lis)/Dulius Iulius (cf. fig. 6) ${ }^{121}$.

Cette inscription fait partie d'un monument de granite $(0,87 \mathrm{~m}$ x $0,38 \mathrm{~m}$ ) localisé dans l'église de Tejeda de Tiétar et qui est désignée par la population locale sous l'appellation la Muerte Pelona. Sa particularité réside dans son texte ambigu et sa décoration anthropomorphe en relief qui occupe les $2 / 3$ inférieurs de la pièce. Toutes les tentatives de transcription ont livré des textes divers, en particulier concernant le théonyme contenu dans l'inscription ${ }^{122}$. La dernière proposition, celle de J. M. Domínguez Moreno, nous semble intéressante dans la mesure où cet auteur fait référence à des divinités préromaines dont l'étymologie peut être rapprochée de celle de Selu, théonyme qui serait attesté à Ibahernando, dans la province de Cáceres. Quant aux Duillae, ce sont des déesses protectrices de la végétation dans le monde celte ${ }^{123}$. En ce qui concerne la figure anthropomorphe, il pourrait s'agir d'un individu, peut-être armé, exécutant une danse rituelle comme cela se pratiquait dans diverses régions de la péninsule dans l'Antiquité ${ }^{124}$. En Estrémadure précisément, mais aussi dans d'autres régions, certains rites (danses, sauts...) sont parfois considérés comme des survivances d'un culte païen de la fertilité agricole et de la virilité ${ }^{125}$. Or, un de ces rites aurait consisté en la lapidation de la pièce qui nous intéresse. J. M. Domínguez Moreno interprète ce geste comme la survivance d'une antique pratique de sacrifice humain à des fins de fertilité agraire ${ }^{126}$.

121 J. M. Domínguez Moreno, El ara votiva de Tejeda de Tiétar y su información sobre una danza prerromana, Norba, VIII-IX, 1987-1988, pp. 33-41.

122 V. Soria SÁnchez, Descubrimientos arqueológicos en Extremadura, REE, XXXI-XXXII, 1975, pp. 204 ss.

123 BlázQuez, Diccionario, pp. 90, 92; J. POKORNY, Indogermanisches Etymologisches Wörterbuch, Berne, 1959-1960, p. 243; SOLANA Y HERNÁNDEZ, Religión y sociedad, p. 156.

124 J. M. BlázQuez, Música, danza, competiciones e himmos en la Hispania antigua, Bellas Artes, VII, 51, 1976, pp. 3-10; F. FrAZER, La rama dorada, Mexico, 1979, p. 52; J. Caro Baroja, El estío festivo, Madrid, 1984, p. 214; Strabon, III, 3, 7; III, 4, 16; Diodore de Sicile, V, 34, 5; Silius ItAlicus, III, 345-349; Appien, Iber. LXXII.

125 J. M. Domínguez Moreno, Cultos a la fertilidad en Extremadura, Mérida, 1987, pp. 7 ss.; on consultera également M. MAYER et I. RODÁ, Les divinités féminines de la fertilité en Hispania pendant l'époque romaine, dans J. BonANNo (éd.), Archaeology and fertility cult in the ancient Mediterranean, Amsterdam, 1986, pp. 293-304.

126 J. M. Domínguez Moreno, art. cit., pp. 39-40. 
Ainsi, on peut être tenté de penser que ce document a remplacé, face à l'interdiction romaine des sacrifices humains, une coutume consistant à sacrifier des êtres humains ${ }^{127}$. Il n'est malheureusement pas possible de préciser la chronologie de ce document en raison de l'état défectueux de la pièce, mais il est possible qu'elle puisse dater des débuts de la domination romaine, à une époque où les Romains imposent leur autorité et font cesser des pratiques qu'ils jugent inadmissibles ${ }^{128}$. Deux autels de Palencia consacrés à Duillis datent, quant à eux, du Ier siècle ap. J.C. ${ }^{129}$.

- TOGA: Togae/Veienta/Tusca/pro/victoria/Garici/Fuscinil $/ v$ (otum) s(olvit) l(ibens) a(nimo) ${ }^{130}$.

Togae/Rufus/Vegetif. v(otum) l(ibens) a(nimo) s(olvit) ${ }^{131}$.

Nous retrouvons au sud des montagnes centrales, à Torre de la Mata (San Martín de Trevejo), et à Valverde del Fresno, une divinité également vénérée à proximité de la sierra de Gata mais dans la province de Salamanque. La mention pro victoria de la première inscription inciterait à penser qu'il s'agit d'une divinité guerrière; en tout cas d'une divinité protectrice adorée dans la région de la sierra de Gata mais aussi dans la région montagneuse de Marvão et San Vicente (pays lusitanien) ${ }^{132}$. J. de Alarcão aurait tendance à considérer Toga comme une divinité vettonne introduite en pays vetton ${ }^{133}$.

- TRITIAECIUS: Deo/Tritialecio Fr/onto F[l]/accinilf. p(osuit) [e] $x$ v(oto) (4).

L'inscription provient de Torremenga, dans le nord-est de la province de Cáceres. Selon C. Callejo Serrano, Tritiaecius serait le dieu éponyme d'un site, Tritia, localisé dans la ferme voisine de Torrejonas ${ }^{134}$.

127 Une telle interprétation mérite toutefois d'être envisagée avec circonspection; la lapidation de la stèle peut également être interprétée comme un geste chrétien de refus d'une sculpture jugée païenne.

128 Plutarque, Quaest. Rom., LXXXIII.

129 Alonso et CRespo, Las manifestaciones, pp. 39-40 n. ${ }^{\circ} 38$ et 39.

130 Melena, Salama, p. 487 n. ${ }^{\circ}$ 11-2; CIL II, 801; BlázQUEZ, Religiones, p. 127; BLÁZQueZ, Diccionario, p. 173.

131 M. García de Figuerola, Inscripción a Toga en la sierra de Gata, FE, 12, 1985, n. $^{\circ} 49$.

132 Olivares, El panteón, pp. 103-105.

133 AlarCão, Novas perspectivas, p. 318.

$134 A E, 1965,74$. 
Nous livrons enfin les trois théonymes suivants avec la plus grande prudence:

- ECUSSUA?: Ecussu[e]?/Mailo/nus Leluri a(ram) p(osuit)/ ll(ibens) a (nimo) ${ }^{135}$.

Retrouvée à Villamiel, le théonyme de cet autel votif demeure extrêmement incertain du fait d'un martelage manifeste, peut-être dans le contexte de la christianisation; nous avons malgré tout retenu la transcription de J. L. Melena. On peut néanmoins se demander s'il ne s'agirait pas d'une variante locale du dieu indigène Cosus, assimilé à Mars ${ }^{136}$.

- [R?]UDINUS OENUS?: [R?]udino/Oeno? A/dercia A/mbati f./v(otum) s(olvit) l(ibens) m(erito) ${ }^{137}$.

Cet autel de Monroy nous livre un théonyme trés hypothétique inconnu par ailleurs; il pourrait s'agir d'un dieu guerrier à en juger par le syncrétisme que constitue Mars Rudianus ${ }^{138}$.

- VORTIACIUS?: Vortialcio? Aviltus [...]/tici(us) Av/ici(us) Tonc(i)/f(ilii) patris/sui v(otum) s(olverunt)/[a(nimo)] l(ibens) ${ }^{139}$.

Montehermoso aurait livré ce théonyme (?) par ailleurs connu au Portugal.

135 C. Callejo Serrano, Fichas de arqueología extremeña, AEA, XXXVI, 1963, 107-108, p. 227.

136 C. Callejo Serrano, Aportación a la epigrafía romana del Campo Norbense, BRAH, CLVII, 1965, pp. 74 ss, pl. XXXIV; MelenA, Salama, p. 489 n. ${ }^{\circ}$; HEp, 1, 1989, 206; J. V. Madruga Flores et J. Salas Martín, A propósito de teónimos indígenas en el conventus Emeritensis, ETF (Serie II), VIII, 1995, pp. 338-339, proposent une transcription différente: Eci[r?]icu.

137 Cossua, variante locale de Cosus, est en effet attesté plusieurs fois dans la région nord-occidentale du Bierzo; le théonyme de Villamiel ne pourrait-il être, de la même façon, une autre adaptation locale de Cosus? Sur Cossua $c f$. Tranoy, Galice, p. 297 et J. M. BlázQueZ, Nuevos teónimos hispanos. Addenda et corrigenda V, Cuadernos de Prehistoria y Arqueología, XIII-XIV, 1986-1987, p. 50 n. ${ }^{\circ} 22$.

138 J. M. Iglesias Gil et J. L. SÁnChez AbAL, Nuevas estelas romanas de la provincia de Cáceres, AEA, L-LI, 1977-1978, p. 424.

139 Sur Mars Rudianus, $c f$. A. GARcía y Bellido, Cantabria romana, Santander, 1952, p. 34; sur le dieu indigène Erudinus, cf. J. M. IGLESIAS GIL, Onomástica prerromana en la epigrafía cantabra, Santander, 1974, pp. 37 ss. 


\section{Dans la regio Turgaliensis}

La partie sud-orientale de la province de Cáceres avait pour centre de rayonnement Turgalium et apparaît comme imprégnée d'un caractère moins marqué de traits indigènes que le nord de la Vettonnie ${ }^{140}$. Mais il ne semble pas que la religion indigène y ait pour autant laissé moins de traces que dans le reste du pays vetton, à en juger par la liste des théonymes que nous livre l'épigraphie de cette région ${ }^{141}$ :

- AENIDIVIS?: Aenidivi?/Varia Lel[...]ntis f./votu(m)/solvit ${ }^{142}$.

Provenant de Zorita, cette inscription présente des problèmes de transcription relevés par C. Callejo Serrano et qui rendent le théonyme Aenidivis assez hypothétique.

- AERBIN[...]: Aerbin[...]/C(aius) Sem/proni/us? Avitu/s a(nimo) l(ibens) $v$ (otum) s(olvit) ${ }^{143}$.

L'autel proviendrait de Salvatierra de Santiago bien que la déesse Erbina apparaisse comme la divinité protectrice des Igaeditani ${ }^{144}$ - AERVIS?: A[...] C[...]/Aervi?/T[...]u. Ti? ${ }^{145}$.

L'état de la pierre, localisée à Ibahernando, est très mauvais mais le théonyme Aervis renverrait plutôt à Aerbin [...], plus fiable ${ }^{146}$.

- AIVOSSIVAEIUS?: Aiv/ossi/vaeio?/Caev/us Li/isai (filius) ${ }^{147}$.

L'autel, endommagé, provient de Plasenzuela mais J. M. Blázquez reconnaît que cette lecture, donnée par M. Roso de Luna, n'est pas sûre.

- ATAECINA: [D(eae) D(ominae) S(anctae)?] Turibr[igensi)?/ /[Ad?]aegin[ae] ${ }^{148}$.

140 HEp, 4, 1994, 242.

141 J. A REDOndo Rodríguez, Algunas consideraciones acerca de la romanización de los Vettones en el sureste cacereño, Norba, V, 1984, pp. 69-79 (= REDONDO, Algunas consideraciones).

142 J. A. REDONDo RodríGuez, Catálogo epigráfico latino de Trujillo y su partido judicial, thèse dactylographiée, Cáceres, 1983.

143 C. Callejo Serrano, Nuevo repertorio epigráfico de la provincia de Cáceres, AEA, XLIII, 1970, p. 155 n. 17 et fig. XVI (= CALlEJo, Nuevo repertorio). 144 AE, 1955, 30; BLÁzQUEZ, Religiones, p. 205; SALINAS, La religión indígena del oeste, p. 334 n. ${ }^{\circ}$; Hep, 5, 1995, 242.

145 B.G. FERnÁNDEZ-AlbAlat, La diosa Erbina, la soberanía guerrera femenina y los límites entre Igaeditanos y Vetones, Conimbriga, XXXII-XXXIII, 1993-1994, pp. 338-401.

146 REDONDO, Regio Turgaliensis, II, p. 593 n. 346.

147 B.G. FERnÁNDEZ-AlbALAT, art. cit., p. 389.

148 M. Roso, Nuevas inscripciones romanas del campo norbense, BRAH, XLVII, 
Do[mi]na[e T]/urib[ri(gensi)] Add[aeg?]/ina[e]/Iul(ius). [B]/adi[us] / v(otum) s(olvit) [l(ibens) a(nimo)] ${ }^{149}$.

Viriatus/A(taecinae) a(ugustae)? l(ibens) d(e)/suo p(onendum) c(uravit) ${ }^{150}$.

Daeae Sanctae/Turibrige(nsi)/P(ublius) Florius/Venustus/l(ibens) a(nimo) posuit (cf. fig. 7) ${ }^{151}$.

Sanctae s/acr(um) Cras/tena/Vitallis ex v(oto)/a(nimo) l(ibens) posuit ${ }^{152}$.

T(itus) Norban(us) T(iti) (filius) Qui(n)tu(s) (At)a(e)cina(e) $\operatorname{ara}(m)$ p(osuit) $v$ (otum)? ${ }^{153}$.

Ces six inscriptions (provenant de Salvatierra de Santiago Ibahernando, Herguijuela et Santa Ana) ont été sans doute érigées en l'honneur d'Ataecina, également désignée sous le nom de Dea Sancta (Turibrigensis), divinité celtique des Enfers, identifiée à Proserpine, et dont l'aire de culte s'étendait jusqu'au Norique ${ }^{154}$.

- BANDUA ROUDEACUS: Bandue/Roudealco a(nimo) l(ibens) p(osuit) ${ }^{155}$.

A Madroñera, des travaux de labour ont ramené à la surface du sol cet autel dont la lecture ne semble pas poser problème.

1905, p. 190 n. 2 (= Roso, Nuevas inscripciones); C. CAllejo Serrano, Cédulas epigráficas del campo norbense, Zephyrus, XVIII, 1967, p. 109; BLÁZQUEZ, Diccionario, p. 25; C. Callejo Serrano, Teónimos falsos en Lusitania, dans Homenaje a Saenz de Buruaga, Badajoz, 1982, pp. 332-334, fait le point sur ce théonyme qu'il considère comme faux.

149 HEp, 6, 1996, 241; J.V. Madruga Flores et J. SAlas Martín, art. cit., pp. 331-335 n. ${ }^{\circ} 2$.

150 Ibid., pp. 335-338.

151 SALinas, La religión indígena del oeste, p. $336 \mathrm{n} .^{\circ} 19$.

152 Roso, Nuevas inscripciones, p. 131 n. ${ }^{\circ} 34$; CALleJo, Nuevo repertorio, pp. 144-145; REDONDO, Regio Turgaliensis, II, p. 594 n. ${ }^{\circ} 344$.

153 CAllejo, Nuevo repertorio, pp. 146-147 n. ${ }^{\circ} 10$ (un $D$. au début de l'inscription a pu exister); SALINAS, La religión indígena del oeste, p. 336 n. ${ }^{\circ} 19$.

154 BlázQuez, Religiones, p. 144; Salinas, La religión del oeste, p. 336 n. ${ }^{\circ} 19$.

155 Sur Ataecina, cf. CAllejo, Nuevo repertorio, p. 145; BlÁZQUEZ, Diccionario, pp. 39-42; R. LóPEz MELERo, Nueva evidencia sobre el culto de Ategina: el epigrafe de Bienvenida, dans Manifestaciones religiosas en la Lusitania. (Primeras Jornadas sobre Manifestaciones religiosas en la Lusitania, (Cáceres, 1984), Cáceres, 1986 (= Manifestaciones religiosas), pp. 93-112; J. V. MADRUGA Flores et J. SALAS MARTín, art. cit., pp. 331-338; J. M. BlÁZQuEZ, Religiones indígenas en la Hispania romana (addenda et corrigenda), Gerión, XIV, 1996, pp. 344-350. 
- BARAECUS? ou REVVE ANABARAECUS?: Baraeco?/ /Nor(banus) ac?/Iunius aed(iles)?/II vir(i) c(...) I(...)?/pro salute ${ }^{156}$. Cette inscription, provenant de Trujillo même, présente une lecture très incertaine et a été l'objet de transcriptions sensiblement différentes ${ }^{157}$. En fait, il pourrait s'agir de [ Revve Ana]baraecus à la première ligne ${ }^{158}$.

- DEIA [...]: Loucin/us Cili f./a(ram) posuilt Deiael[...]/[...] ${ }^{159}$.

A Ibahernando, on mis au jour cet autel consacré à Deia [...]; le nom précis de la déesse devait suivre à la cinquième et peut-être à la sixième ligne mais la transcription de $\mathrm{R}$. Hurtado est bien peu fiable pour pouvoir proposer un nom ou une épithète, pas même Sanctae Turibrigensis. On remarquera l'orthographe Deiae au lieu de Deae. Ne s'agirait-il pas de la véritable appellation de la déesse ou bien d'une déformation de Deae ${ }^{160}$ ?

- EADURUS [...]AECUS?: [...Do]/quiri f. E/aduro/[...]aec/o a(nimo) l(ibens) $/ v$ (otum) $[s(\text { olvit })]^{161}$.

Selon J. A. Rodríguez, nous serions bien en présence d'un autel érigé en l'honneur de Eadurus Baraecus et non Eaduroeaecus, théonyme défendu par M. Beltrán Lloris. Il semble bien que quelques lettres aient été effacées devant aeco mais celles-ci sont difficilement identifiables; ainsi, d'autres théonymes sont envisageables: Vorteaecus, Roudeaecus...

- NABIA: [...]/[...]urus T/ancini l(ibertus)/Nabi(e) s(acrum) a(nimo) l(ibens)/aram p/osuit et/vot(um) sol(vit) ${ }^{162}$.

156 Beltrán, Aportaciones, pp. 92-93 n. ${ }^{\circ}$ 64; AE, 1977, 432.

157 CIL II, 5276; REDONDO, Regio Turgaliensis, II, p. 594 n. ${ }^{\circ} 349$.

$158 C f$. également BlázQueZ, Religiones, pp. 74-75; HEp, 4, 1994, 253 et HEp, 6, 1996, 250; sur Revve Anabaraecus, cf. A. M. Canto, A. Bejarano et F. Palma, El mausoleo del dintel de los ríos de Mérida, Revve Anabaraecus y el culto de la confluencia, MM, 38, 1997, pp. 247-294 (= CANTO et alii, Revve Anabaraecus).

159 C. Callejo Serrano, Fichas de arqueología extremeña, AEA, XXXVI, 1963, 107-108, p. 225.

160 Tranoy, Galice, p. 274, cite Daea Sancta de Vila Chã: il s'agit d'une déformation de Dea Sancta c'est-à-dire Ataecina.

161 Redondo, Regio Turgaliensis, II, p. 595 n. 351 (Eaduro Baraeco); BeLTRÁN, Aportaciones, p. 89 n. ${ }^{\circ}$ 61, (Eaduroaeco).

162 J. L. Melena, Un ara votiva romana en El Gaitán, Cáceres, Veleia, I, 1984, p. 234, qui réfute la transcription ABISAI; OLIVARES, El panteón, p. 117. 
L'autel retrouvé à Trujillo même, présente des lettres du IIIème siècle ap.J.C.

- NETO?: Netoni/deo/Caelius/v(otum) s(olvit) l(ibens) a(nimo) ${ }^{163}$.

A Trujillo, on a également retrouvé cette inscription consacrée au dieu guerrier Neto, souvent associé à Mars. Ce document confirme donc le texte de Macrobe sur l'existence du culte de ce dieu dans la péninsule ${ }^{164}$.

- REVVE ANABARAECUS: Revve Analbaraeco/Afer Albinilf. Turolus $/ v$ (otum) s(olvit) l(ibens) $m$ (erito $)^{165}$.

Cette inscription proviendrait de Ruanes mais Orense est parfois avancé comme étant son lieu d'origine ${ }^{166}$. Revveana Baraecus serait-il un théonyme dérivé de Reva, autre divinité guerrière mentionnée notamment dans l'inscription de Cabeço das Fráguas ${ }^{167}$ ? Quant à l'épithète, elle est déjà connue en tant que théonyme et, selon A. Holder, pourrait être mise en relation avec la notion de colère, de fureur ${ }^{168}$. Cependant, récemment on a émis l'hypothèse que Revve Anabaraecus serait en relation avec le culte de la confluence (Ana et Baraecus étant deux cours d'eau) ${ }^{169}$.

- ROUDAECUS: [...] Roud/aeco v(otum) s(olvit) l(ibens) ${ }^{170}$.

Cet autel a été retrouvé à la confluence de deux cours d'eau, à Villavieja (Trujillo), en compagnie de nombreux autres documents épi-

163 CIL II, 5278; FernándeZ-Albalat, Guerra y religion, p. 331; Olivares, El panteón, p. 112 qui insiste sur le doute pesant sur ce document et propose Netoniceo; on lira également ENCARNAÇão, Divindades, pp. 248-250.

164 Macrobe, Sat., I, 19, 5; d'autres théonymes proches de Neto existent en Celtibérie, $c f$. F. Marco Simón, La religión de los Celtíberos, dans Simposium sobre los Celtíberos, I (Daroca, 1986), Saragosse, 1987, p. 63.

165 CIL II, 685; SALinas, La religión indígena del oeste, p. 339 n. ${ }^{\circ} 32$; CANTO et alii, Revve Anabaraecus, p. 277.

166 Redondo, Regio Turgaliensis, II, p. 595 n. ${ }^{\circ} 353$ (Ruanes); Tranoy, Galice, p. 285 n. ${ }^{\circ} 190$.

167 Sur Reva, cf. BlázQuez, Religiones, pp. 185-187; BlázQUEZ, Diccionario, p. 141; FernándeZ-Albalat, Guerra y religión, pp. 311-324; F. Villar, El teónimo lusitano Reve y su epíteto, in MeID (W.) et ANREITER (P.) (éd.), Die grösseren alteltischen Sprachdenkmäler, Wiesbaden, 1996.

168 A. HoldER, Altceltischer Sprachschatz, I, Leipzig, 1907, col. 119, 350; FERNÁNDEZ-AlBALAT, Guerra y religión, pp. 319-320.

169 CANTO et alii, Revve Anabaraecus, pp. 247-294.

170 AE, 1977, 430; Beltrán, Aportaciones, p. 88 n. ${ }^{\circ}$ 60; Redondo, Regio Turgaliensis, II, p. 595 n. ${ }^{\circ} 354$. 
graphiques dont un autre autel, peu lisible, qui pourrait être une autre dédicace à Roudaecus, et un autel dédié à Eadurus [...]aecus ${ }^{171}$. Il est probable qu'il y avait à cet endroit un sanctuaire.

- SALAMACNABIA?: Salam/acnabi(ae)?/Ilucius/v(otum) s(olvit) l(ibens) a (nimo) ${ }^{172}$.

Provenant de Trujillo, cet autel mentionnerait un ou plusieurs théonymes incertains (Salamacnabia? Salama? Nabia). L'hypothèse d'une seule divinité syncrétique n'est pas à exclure et nous semble plus probable qu'une inscription dédiée à Salama et à Nabia car dans ce dernier cas, il s'agirait d'un exemple unique en Vettonnie d'une seule dédicace à deux divinités indigènes. En fait, si Nabia est incontestable, on peut émettre quelques doutes sur la première partie du théonyme.

- SELU?: Selu? P(ublius)/Caeciliu/s Maxumus l(ibens) a(nimo) s(olvit) ${ }^{173}$.

Provenant d'Ibahernando, cet autel fait mention d'un dieu inconnu ailleurs. La transcription nous semble très incertaine.

- TURCULLA: Turculla/[e] [s]acrum/Norbana/Quintillal la(nimo) l(ibens) p(osuit) ${ }^{174}$.

L'autel a été retrouvé à Puerto de Santa Cruz mais il a été perdu. On peut penser que cette divinité est liée au site vetton de Turgalium.

3. Dans le reste de la Vettonnie

- ARICONA?: Arico/nae?/Alles/Alion/icum/v(otum) a(nimo) l(ibens) s(olvit) (Talavera de la Reina, TO) ${ }^{175}$.

- ATAECINA: Bassus/Turobri(gensis)/eques al/ae Vetto/num ara/m pos(u)it/Ataecin/ae votu/m solvi(t)/[l]iben[s]/[merito] (Caleruela, TO $)^{176}$.

171 Beltrán, Aportaciones, pp. 87-88 n. ${ }^{\circ} 59$ et pp. 89-90 n. ${ }^{\circ} 61 ; H E p, 6,1996$, 242: .../aeco/Calpur(nius, a)/Cleme $(n)$ s.

172 CIL II, 5279; ILER, 921; REDONDO, Regio Turgaliensis, II, p. 595 n. 355.

173 J. RAMÓN Y FERNÁNDEZ OXEA, De epigrafía cacereña, BRAH, CXXXVI, 1955, p. 258; ILER, 927 (qui juge incertain le nom de ce dieu).

$174 A E, 1993,959 ;$ H. Gimeno et A. U. StYlow, Juan Pérez Holguín y la epigrafía trujillana, Veleia, X, 1994, p. 151 n. 43 (= Gimeno et STYLOW, Epigrafía trujillana); HEp, 5, 1995, 231.

$175 A E, 1946,14$; GoNZÁLEZ, Las unidades, p. 122 n. ${ }^{\circ} 17$.

176 AE, 1988, 823; M.P. GonzÁlez-Conde Puente, Bassus Turobrigensis y la inscripción de Ataecina en Caleruela (Toledo), SHHA, VI, 1988, pp. 131-132. 
- TOGOTIS ou TOGOS?: Togoti/L.Vibius/Priscus/ex voto (Monte de San Vicente, TO) ${ }^{177}$.

Nous livrons enfin ci-dessous, avec de plus grandes réserves encore, les deux théonymes suivants:

- DENNA?: C.C./ Mod/estin/us Den/n(a)e? v(otum)/l(ibens) a(nimo) $s$ (olvit) (Las Posadillas, BA) ${ }^{178}$.

- UPAISOUN?: Upaisoun?/Titanus/Vecisus?/Suri f. v(otum)/libens) a(nimo) s(olvit) (Las Posadillas, BA) ${ }^{179}$.

La démarche géographique que nous avons adoptée se trouve justifiée par l'existence de quelques grandes zones. Dans chacune d'entre elle, la religion indigène a adopté des caractères particuliers.

- Dans la province de Salamanque, la fonction des divinités indigènes devait s'articuler assez fortement autour du thème de la nature et en particulier de l'eau. Il est vrai que cette partie nord de la Vettonnie se caractérise par l'existence d'un réseau hydrographique important qui a dû influencer les mentalités religieuses.

- Plus à l'ouest, au Portugal, le panthéon indigène est marqué par l'influence de la Lusitanie toute proche; c'est particulièrement net avec Arentia, et surtout, avec Band et ses dérivés ${ }^{180}$.

- La province d'Ávila se caractérise par la faible trace d'épigraphie votive, Velicus étant le seul dieu formellement attesté dans le sanctuaire de Postoloboso, et nulle part ailleurs en Vettonnie. Ainsi, l'in-

177 CIL II, 983; ILER, 934; BLÁZQUEZ, Religiones, p. 127; BLÁZQUEZ, Diccionario, p. 173; l'inscription ne provient pas de Caesarobriga même mais du sanctuaire de la Virgen del Piélago, à quelques kilomètres au nord de Talavera.

$178 A E, 1986,319$; D. VAquerizo GIL, Epigrafía romana de la "Siberia extremeña”, REE, XLII, 1986, pp. 126-127 n. ${ }^{\circ} 10$.

$179 A E, 1986,320$; D. VAQUERIZO GIL, art. cit., pp. 127-128 n. ${ }^{\circ} 11$; l'auteur ne s'étend pas sur ce théonyme assez douteux.

180 Récemment, J. de AlARCÃo, Novas perspectivas, pp. 293-349, a abordé l'étude de la religion des Lusitaniens dans une perspective géographique et ainsi abouti à une délimitation Lusitaniens/Vettons différente de la notre. Ainsi, la présence de divinités lusitaniennes tels que Quangeius, Arentius/Arentia, Trebarunis à l'est de la serra da Estrela et dans la province de Cáceres (au nord du Tage) incite cet auteur à porter la limite ethnique nettement à l'intérieur de cette province. Nous préferons voir une influence du panthéon lusitanien en pays vetton, à l'est de la serra da Estrela jusqu'à Capera et Caesarobriga et rappeler le caractère instable des limites ethniques en relation avec le mode de vie pastoral des populations tant lusitaniennes que vettonnes; nous renvoyons à notre article: Vettonia Antiqua: les limites ethniques et administratives d'un peuple de l'ouest de la Meseta dans l'Antiquité, SHHA (article sous presse). 
fluence du dieu lusitanien Endovellicus se fait ressentir jusque dans l'est de la Vettonnie.

- Plus au sud, la Haute-Estrémadure, bien que séparée du reste de la Vettonnie par une chaîne de montagnes, semble constituer un prolongement où on retrouve certaines divinités du nord-ouest de la Vettonnie: Toga, présente à proximité et sur les deux versants de la sierra de Gata, Quangeius et Bandis? Vort[ia.]cius? présents à Malpartida de Plasencia.

- Dans le sud vetton, la regio Turgaliensis apparaît comme davantage à l'écart: à l'exception de Bandua, aucune des autres divinités ne se retrouve ailleurs en Vettonnie; le culte d'Ataecina y semble bien implanté ainsi que celui de Revve Anabaraecus (à Ruanes et à Trujillo même), par ailleurs attesté à Augusta Emerita dont la regio Turgaliensis était une préfecture.

- Les cités de Lacimurga et de Caesarobriga ont révélé très peu de cultes indigènes: Denna? et Upaisoun? dans la première de ces cités, Togotis? Ataecina, Aricona à Talavera de la Reina.

Ainsi, on a la très nette sensation, malgré quelques divinités au culte un peu plus diffus, d'un manque d'homogénéité, d'un cloisonnement qui se traduit par l'existence de cultes locaux dont le nombre originel est à peine suggéré par les quelques témoignages épigraphiques dont nous disposons pour la Vettonnie. Quelques thèmes, comme celui de la guerre, de la souveraineté guerrière (Bandis, Toga, Roudeacus, Neto) de la fertilité (Velicus), de la montagne (Salama) transparaissent néanmoins ${ }^{181}$. On notera par ailleurs que tous ces thèmes peuvent apparaître comme liés à l'idée d'élevage, et plus précisément au taureau ${ }^{182}$. Symbole de force, de combativité, de victoire et de souveraineté guerrière, le taureau, matérialisé sous l'aspect de sculptures de granite, peut être mis en relation avec une partie de ces divinités mais sans pour autant être leur représentation; il pouvait symboliser la puissance et la souveraineté d'individus socialement privilégiés voire de

181 Sur les divinités liées à la guerre et que l'on retouve en Vettonnie, $c f$. FERNÁNDEZ-AlBALAT, Guerra y Religión, pp. 23-52, 155-179, 280-284, 331-332.

182 FERnÁNDEZ-Albalat, Guerra y religión, p. 280, évoque le rôle du taureau dans la symbolique celtique mais curieusement sans évoquer les sculptures de taureau sinon les représentations de têtes de taureau sur certains autels votifs et la statuette retrouvée dans les Pyrénées représentant un guerrier dont la cuirasse comporte une figure de taureau et le casque à trois cornes; rien de comparable n'a été retrouvé en Vettonnie si bien qu'on peut se demander si ce symbole du taureau existait en pays vetton. 
communautés particulières, de "confréries de guerriers" pour reprendre l'expression de M. B. García Fernández-Albalat ${ }^{183}$.

En fait, au-delà de la thèse de Fernández-Albalat, il s'agit de mieux cerner le milieu social des dédicants.

\section{Divinités Indigènes et Société}

\section{SALAMANQUE}

\begin{tabular}{|c|c|c|c|c|c|}
\hline \multirow[t]{2}{*}{ Divinités indigènes } & \multicolumn{3}{|c|}{ Dédicants } & \multicolumn{2}{|c|}{ Filiation } \\
\hline & Ind. & Rom. & T.N. & Ind. & Rom. \\
\hline ACPULSOIUS? & + & & & & + \\
\hline CAESARICIAECUS & + & & & + & \\
\hline CANTUNAECUS & $+?$ & & & + & \\
\hline CELIBORCA & + & + & & & \\
\hline FAVILIUS? & & + & & & \\
\hline ILURBEDA & & + & + & & \\
\hline
\end{tabular}

NATRICUS (ou NABIA)

TOGA ++

183 FERnÁNDEZ-AlBALAT, Guerra y religión, p. 344. 


\section{PORTUGAL}

\begin{tabular}{|c|c|c|c|c|}
\hline \multirow[t]{2}{*}{ Divinités indigènes } & \multicolumn{2}{|c|}{ Dédicants } & \multicolumn{2}{|c|}{ Filiation } \\
\hline & Ind. & Rom. T.N. & Ind. & Rom. \\
\hline \multicolumn{5}{|l|}{ AELVA? } \\
\hline AETIUS & + & & & \\
\hline $\begin{array}{l}\text { ARENTIA EQUO- } \\
\text { TULLAICENSIS }\end{array}$ & & + & + & \\
\hline $\begin{array}{l}\text { ARANTIA OCELAECA et } \\
\text { ARANTIUS OCELAECUS }\end{array}$ & & + & + & \\
\hline BANDIS ARBARIAICUS & + & & + & \\
\hline BANDEIS BRIALEACUS & & + & + & \\
\hline BANDIS ISIBRAIA & + & & + & \\
\hline BANDIS ISIBRAIA & & + & + & \\
\hline BANDIS VORTEAECEUS? & + & & + & \\
\hline VORTIAECIUS? & + & $+\quad+$ & & \\
\hline CORVA & & $+?$ & & \\
\hline LAEPUS & & + & & + (Patr.) \\
\hline LAEPUS & + & & + & + \\
\hline LAEPUS & & + & + & \\
\hline LANEANA? & + & & & \\
\hline M.B.S. & & + & & \\
\hline QUANGEIUS & + & & + & \\
\hline QUANGEIUS & + & & + & \\
\hline QUANGEIUS & $?$ & & $?$ & \\
\hline R.C. & + & & + & \\
\hline REUS PARAMAECUS? & & + & $?$ & \\
\hline$[\ldots]$ AMOI? & + & & + & \\
\hline
\end{tabular}

Conimbriga, 41 (2002) 63-103 


\section{ÁVILA}

\begin{tabular}{|c|c|c|c|c|}
\hline \multirow[t]{2}{*}{ Divinités indigènes } & \multicolumn{2}{|c|}{ Dédicants } & \multicolumn{2}{|c|}{ Filiation } \\
\hline & Ind. & Rom. T.N. & Ind. & Rom. \\
\hline DEUS TOGOTIS? & & + & & \\
\hline \multicolumn{5}{|l|}{ MAIDUANA } \\
\hline VAELICUS & + & & + & \\
\hline VAELICUS & + & & + & \\
\hline VELICUS & + & & & \\
\hline DEUS VELICUS & & $+\mathrm{G}$ & & \\
\hline DEUS VELICUS & $+?$ & & & \\
\hline VELICUS & & + & & \\
\hline \multicolumn{5}{|l|}{ D.V. } \\
\hline \multicolumn{5}{|l|}{ D.V. } \\
\hline$?$ & + & & & \\
\hline \multicolumn{5}{|l|}{ V[AELI?]CUS } \\
\hline DEUS VELICUS & + & & & \\
\hline D.V.S & & & & \\
\hline
\end{tabular}


HAUTE ESTREMADURE

\begin{tabular}{|c|c|c|c|c|}
\hline \multirow[t]{2}{*}{ Divinités indigènes } & \multicolumn{2}{|c|}{ Dédicants } & \multicolumn{2}{|c|}{ Filiation } \\
\hline & Ind. & Rom. T.N. & Ind. & Rom. \\
\hline \multicolumn{5}{|c|}{$\begin{array}{l}\text { ARABOCOROBA?EICOBUS? } \\
\text { TALUSICUS? }\end{array}$} \\
\hline ASITRITA & $+?$ & + & + & \\
\hline \multicolumn{5}{|l|}{ ASITRITA RAPHICIOGISA? } \\
\hline BANDIS? VORT[IA]CIUS & + & & & \\
\hline QUANGEIUS? & + & & + & \\
\hline ECUSSUA? & + & & + & \\
\hline PALANTICUS & + & & + & \\
\hline [R?]UDINUS OENUS? & + & & + & \\
\hline SALAMA & & + & & \\
\hline SALAMA & + & & + & \\
\hline SELLAES DUILLAS? & + & + & & \\
\hline TOGA & & + & & \\
\hline TOGA & & + & & + \\
\hline TRITIAECIUS & & + & & + \\
\hline VORTIACIUS? & + & & + & \\
\hline
\end{tabular}

Conimbriga, 41 (2002) 63-103 


\section{REGIO TURGALIENSIS}

\begin{tabular}{|c|c|c|c|c|c|}
\hline \multirow[t]{2}{*}{ Divinités indigènes } & \multicolumn{3}{|c|}{ Dédicants } & \multicolumn{2}{|c|}{ Filiation } \\
\hline & Ind. & Rom. & T.N. & Ind. & Rom. \\
\hline AENIDIVIS? & & + & & ? & \\
\hline AERBIN $[\ldots]$ & & + & + & & \\
\hline
\end{tabular}

AERVIS?

\begin{tabular}{|c|c|c|c|c|}
\hline ATAECINA $^{184}$ & & & & \\
\hline ATAECINA $^{184}$ & & + & & \\
\hline ATAECINA $^{184}$ & + & & & \\
\hline ATAECINA $^{184}$ & & + & + & \\
\hline ATAECINA $^{184}$ & $+?$ & & & + \\
\hline ATAECINA $^{184}$ & & + & + & + \\
\hline BANDUA ROUDEACUS & & & & \\
\hline $\begin{array}{l}\text { BARAECUS? ou } \\
\text { REVVE BARAECUS? }\end{array}$ & & + & & \\
\hline DEIA $[\ldots]$ & + & & & + \\
\hline EADURUS [...]AECUS? & & & & + \\
\hline NABIA & $+?$ & & & $+($ patron $)$ \\
\hline NETO? & + & & & \\
\hline REVVE ANABARAECUS & & + & & + \\
\hline
\end{tabular}

\section{ROUDAECUS}

SALAMACNABIA? +

\begin{tabular}{lll}
\hline SELU? & + & + \\
\hline TURCULLA & + \\
\hline
\end{tabular}

184 Nous livrons ici la dénomination la plus usuelle de cette divinité étant donné l'incertitude pesant sur la transcription des diverses dénominations dans la zone de Trujillo. 
CIVITATES CAESAROBRIGENSIS ET LACIMURGENSIS

\begin{tabular}{|c|c|c|c|c|c|}
\hline \multirow[t]{2}{*}{ Divinités indigènes } & \multicolumn{3}{|c|}{ Dédicants } & \multicolumn{2}{|c|}{ Filiation } \\
\hline & Ind. & Rom. & T.N. & Ind. & Rom. \\
\hline ARICONA? & + & & & & \\
\hline ATAECINA & & + & & & \\
\hline TOGOTIS (ou TOGOS)? & & + & + & & \\
\hline DENNA? & & + & & & \\
\hline UPAISOUN? & + & & & + & \\
\hline
\end{tabular}

Nous pouvons constater assez aisément que les divinités indigènes sont avant tout adorées par des individus qui se caractérisent par un enracinement certain dans le milieu indigène rural, même si leur onomastique est en partie latine. Ce phénomène se retrouve, avec quelques nuances, dans l'ensemble de la Vettonnie.

Dans la province de Salamanque, les dédicants portant un nom indigène et ceux portant un nom latin sont en nombre presque égal. Une divinité comme Ilurbeda est adorée par un citoyen romain ne présentant aucun élément nominal d'origine indigène $(Q$. Manilius $F a$ cundus). A Salvatierra de Santiago C. Sempronius Avitus, qui a élevé un autel à Aerbin [...], est un cas semblable. Il est difficile de préciser l'origine de ces deux citoyens qui n'ont pas indiqué leur filiation; rien n'indique qu'il puisse s'agir d'Italiens. Il est possible que nous soyions également en présence d'indigènes ayant obtenu la citoyenneté romaine et dont les origines ne sont plus décelables dans l'onomastique ${ }^{185}$.

185 BlázQuez, Religión y sociedad, p. 75 n. ${ }^{\circ}$ 8, indique, à propos de la stèle dédiée à Ilurbeda, que "le dédicant est romain" mais rappelle plus loin que "sous des noms typiquement romains se cachent, alors que le second siècle est bien avancé, des gens d'ascendance indigène qui ont abandonné leur onomastique d'origine"; ; 'il s'agit d'Italiens, ils se sont en tout cas bien intégrés au milieu indigène, jusqu'à adopter les dieux vettons et un support épigraphique (texte et caractères externes) de facture indigène; sur l'onomastique des individus ayant fait ériger des autels votifs dans le nord de la Vettonnie on lira avec intérêt M. SALINAS et A. Romero PÉREZ, Onomástica y religión en las provincias de Salamanca y Ávila, SRPE, XXXV-XXXVII, 1995, pp. 13-28. 
En Vettonnie portugaise, on retrouve cette onomastique mixte; les noms indigènes sont à peine plus nombreux que les noms latins. Des divinités très ancrées dans le milieu local, comme Arentia Equotullaicensis ou Bandeis Brialeacus, sont l'objet d'un culte même de la part d'individus, portant un nom romain. En revanche, aucun tria nomina, à l'exception de Flavius Turius Felix, n'apparaît dans les dédicaces de cette région, sans doute en raison de l'éloignement des villes. On note enfin que la plupart des noms des pères sont indigènes: la romanisation de l'onomastique a donc peu d'influence sur la pratique de la religion indigène.

Dans la province d'Ávila, les autels votifs de Postoloboso se caractérisent par le faible usage (romain) de la filiation dans les dédicaces, y compris dans celles des indigènes au nom latin, soit environ la moitié des dédicants, ce qui peut apparaître comme important dans un sanctuaire indigène comme celui de Postoloboso où par ailleurs les structures suprafamiliales sont très bien réprésentées. On peut être également surpris de noter la présence dans ce contexte d'un nom grec (Helene) sur un des autels dédiés à Velicus mais rappelons que le dieu Endovellicus avait la particularité d'être adorée par une population ethniquement et socialement très hétérogène ${ }^{186}$. Il est probable, même en l'absence d'un vocabulaire explicite, qu'une partie des dédicants aient été des esclaves (Mirtuo?) ou des affranchis (Marcia Helene?), ce qui pourrait expliquer le faible usage de la filiation, voire l'absence de noms de dédicants sur certains autels. Il faut néanmoins rappeler que Marcia Helene semble le seul anthroponyme en partie grec que l'on puisse mettre en relation, en Vettonnie, avec un culte indigène.

En Haute-Estrémadure, on retrouve les mêmes caractéristiques générales que dans le nord de la Vettonnie. On ne recense aucun tria nomina, à une seule exception près (Avitus [...]ticius Avicius) mais la pratique de la filiation est majoritaire, y compris pour les dédicants au nom indigène. On remarquera que Toga, présente sur deux autels, est adorée dans les deux cas par des individus au nom latin et l'un d'eux est le seul dans toute la région à comporter deux éléments nominaux

186 BlázQUez, Diccionario, p. 95; KNAPP, Latin Inscriptions, p. 406; A. LOZANO VeliLLA, Los antropónimos griegos y su presencia en los cultos peninsulares, SHHA, VI, 1988, p. 100; id. Antropónimos griegos en la epigrafía religiosa latina. Contribución al estudio sociológico de la religión romana en Hispania, Gerión, VII, 1989, pp. 207-239. 
(Veienta Tusca): il pourrait s'agir d'une épouse de citoyen romain, en tout cas, probablement une personne faisant partie d'un milieu aisé. Le contexte qui émane de l'inscription laisse entendre que Veienta Tusca a pu organiser des combats, d'où cette dédicace "pour la victoire de Garicus Fuscinus".

Dans la région de Turgalium, les noms indigènes l'emportent faiblement sur les noms latins et sur une vingtaine d'autels, seuls Aerbin [...], Ataecina et Selu? ont été honorés par des citoyens romains: $C$. Sempronius Avitus, P. Florius Venustus et P. Caecilius Maximus.

Bien que provenant également du milieu rural, les inscriptions votives retrouvées dans les cités de Caesarobriga et de Lacimurga subissent l'influence des chefs-lieux des cités. Au nord de Talavera de la Reina, Togotis ou Togos? est honoré par un citoyen romain L. Vibius Priscus, qui n'indique cependant ni sa filiation et ni sa tribu. Aux confins des pays vetton et carpétan, un auxiliaire de l'ala Vettonum, Bassus, a érigé un autel en l'honneur d'Ataecina ${ }^{187}$. L'autel consacré à Denna? dans la cité de Lacimurga, a été érigé par un individu, peut-être un autre citoyen ayant étrangement abrégé son nomen ${ }^{188}$.

Ainsi, au terme de cette tentative d' inventaire et d'interprétation des divinités indigènes vettonnes, nous pouvons constater que l'épigraphie votive de ce territoire nous livre un nombre considérable de théonymes indigènes: 82 en prenant en compte les diverses mentions d'un même théonyme et en considérant qu'un théonyme formé de deux éléments constitue un seul nom de divinité. Nous n'avons relevé que les noms de divinités ne comportant aucune influence latine autre que le théonyme lui-même (à quelques exceptions près comme la formule deus précédant parfois le théonyme ou la dénomination Dea Sancta et ses dérivés), laissant deviner qu'il s'agit d'un syncrétisme. Ces théonymes «bruts» apparaîssent parfois, mais c'est rare, sous des formes orthographiques différentes bien que très proches (Vaelico et Velico, Bandis et Bandeis, Arentia et Arantia): il faut sans doute voir là l'influence d'un milieu dédicant fortement imprégné d'indigénisme et les conséquences d'une mise par écrit récente de ces théonymes (certains appa-

187 J. M. ABASCAL, De nuevo sobre Ataecina y Turobriga. Exploraciones del año 1900 en Las Torrecillas (Alcuéscar, Cáceres), AEA, LXIX, 1996, pp. 275-280.

188 D. VAQUERIZO GIL, art. cit., pp. 126-127, envisage plusieurs possibilités pour l'abréviation initiale C.C.; différentes interprétations aussi peu convaincantes les une que les autres. 
raîssent peut-être pour la première fois en Vettonnie sous forme écrite). Tous ces théonymes ne doivent cependant pas faire illusion. Il est vrai qu'ils constituent des témoignages d'une certaine tolérance de la part des autorités romaines (il n'y a là aucune particularité vettonne par rapport à l'ensemble du monde romain) mais ils n'impliquent pas nécessairement la survivance de pratiques et de rites préromains dont nous n'avons pratiquement pas trace sous le Haut-Empire. Aussi, sous des appelations indigènes mais rédigées en latin, ces noms de divinités ne sont-ils pas seulement des résidus linguistiques d'une ancienne religion en train d'être supplantée par les dieux romains? Il est bien difficile, en tout cas de distinguer la part de la religion en déliquescence et la part des cultes indigènes qui constituent des éléments de «résistance» ou de dynamisme culturel dans le contexte de la domination romaine. Finalement, tous ces témoignages théonymiques ne semblent pas devoir être interprétés d'une façon homogène. Il faudrait être davantage renseigné sur la nature de chacune de ces divinités, sur son histoire et sur le milieu social leur vouant un culte afin de pouvoir déterminer avec certitude leur degré de vitalité parmi toutes les divinités qui étaient vénérées par la population vettonne du Haut-Empire. Quelques éléments peuvent néanmoins être avancés avec une relative certitude: ces divinités sont avant tout adorées par des individus qui sont enracinés dans le milieu indigène rural et aucune d'entre elles ne s 'impose à l'ensemble ou même à une partie de la Vettonnie car les différentes mentions d'une même divinité ne sont pas courantes (Toga, Quangeius, Ataecina), les théonymes attestés plusieurs fois à Postoloboso (Vaelicus) et Quinta de São Domingos (Laepus) étant des exceptions car il s'agit là de sanctuaires ou bien d'un atelier dans le cas São Domingos. Cependant, on est loin de constater que les divinités vettonnes aient été totalement rejetées par les individus davantage ancrés dans la romanité. Enfin, on ne peut qu'être frappé par l'abondance des témoignages de cultes indigènes dans toute la partie occidentale de la Vettonnie, tout particulièrement dans les zones à proximité immédiate du pays lusitanien. Il est incontestable que le panthéon lusitanien a eu une influence considérable en Vettonnie (jusqu'à Postoloboso avec le culte de Va(e)licus). En ce sens, on est en droit d'évoquer une réelle communauté de culte entre Lusitaniens et Vettons. 


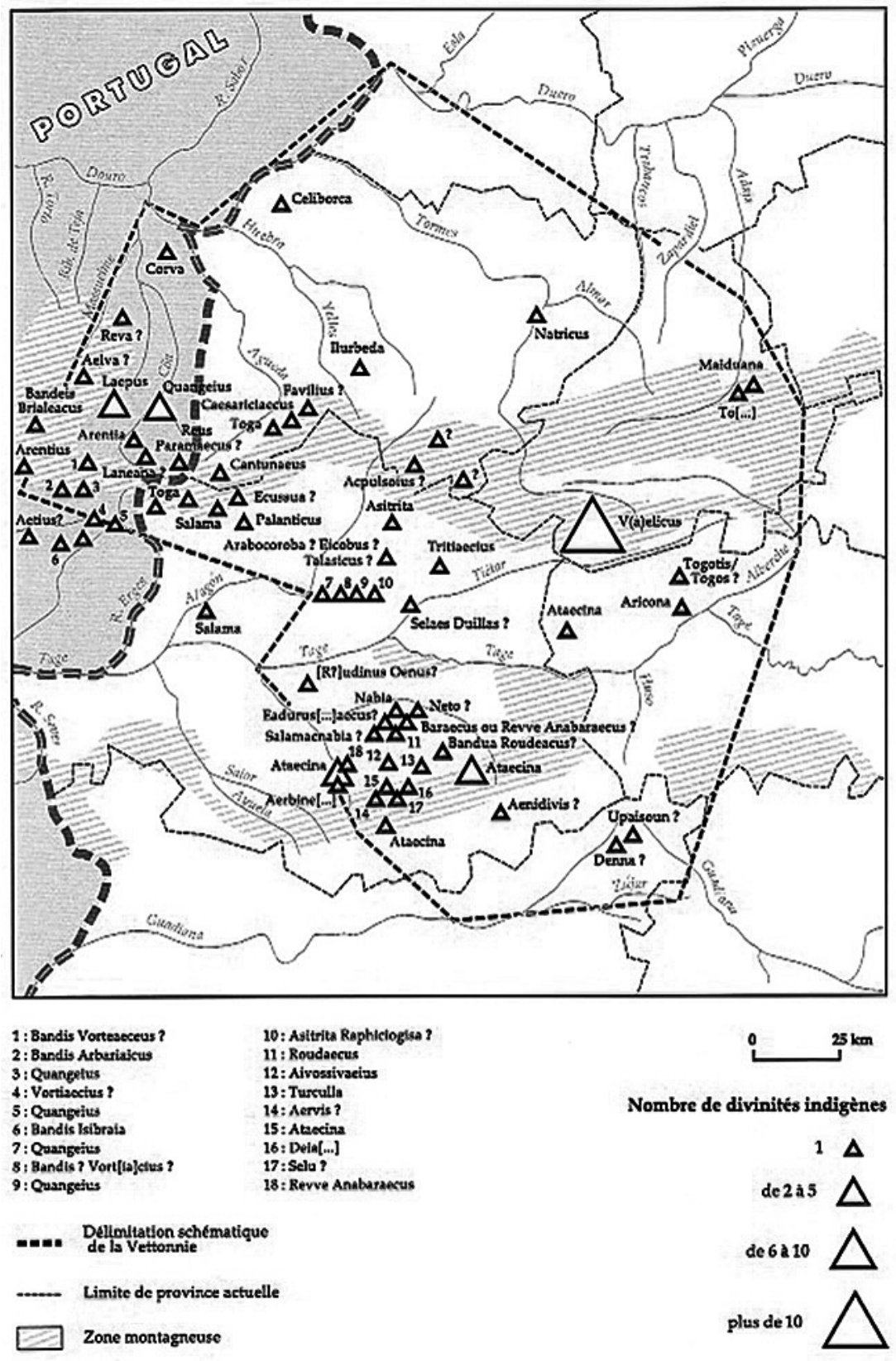

FIG. 1 - Les divinités indigènes en Vettonnie 


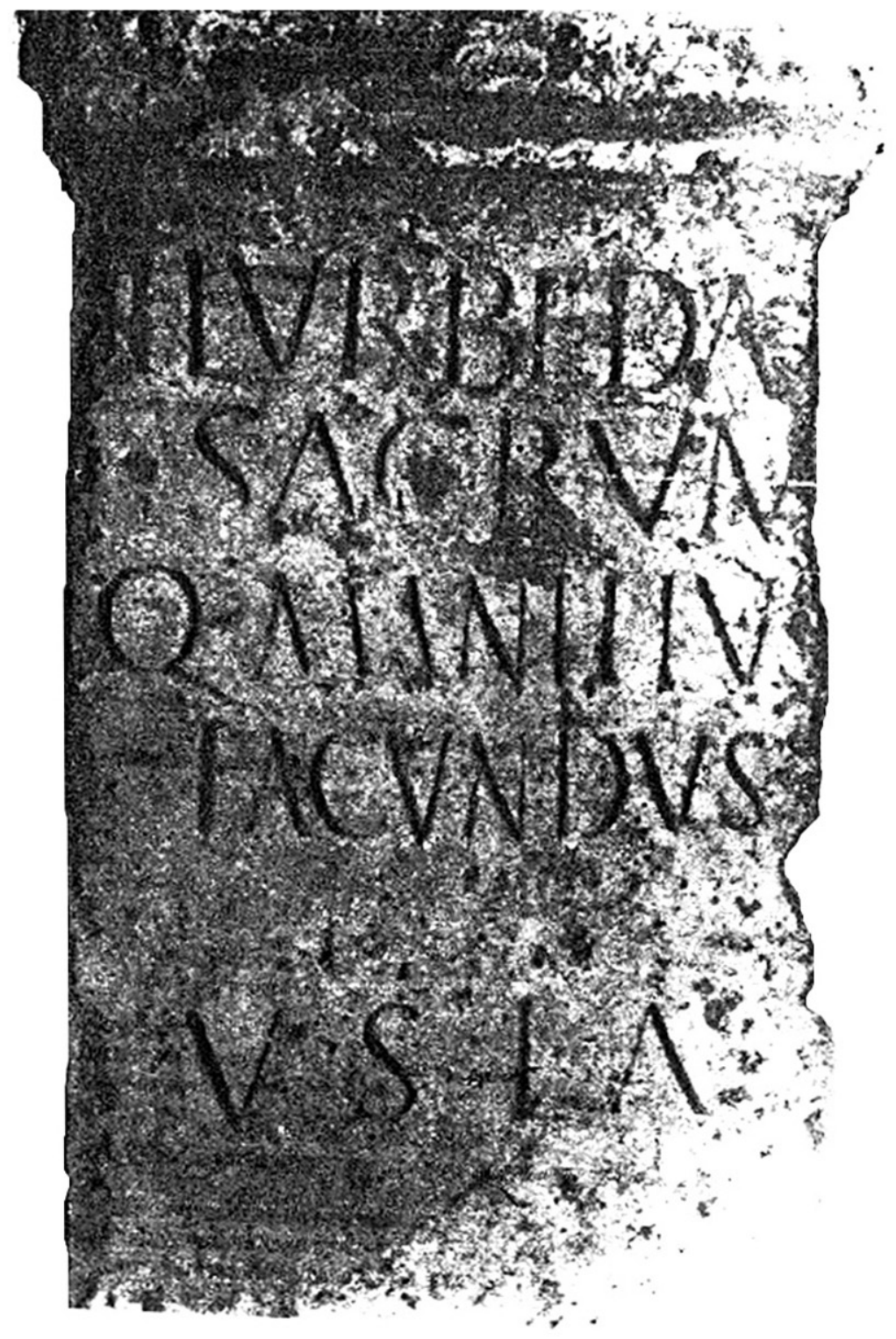

FIG. 2 - Autel consacré à Ilurbeda (Segoyuela, prov. de Salamanque) Dimensions: $0,78 \mathrm{~m} \mathrm{x} \mathrm{0,44} \mathrm{m}$

D'après J. MANGAS, Nuevas inscripciones latinas de Salamanca y provincia, $A E A$, XLIV, 1971 

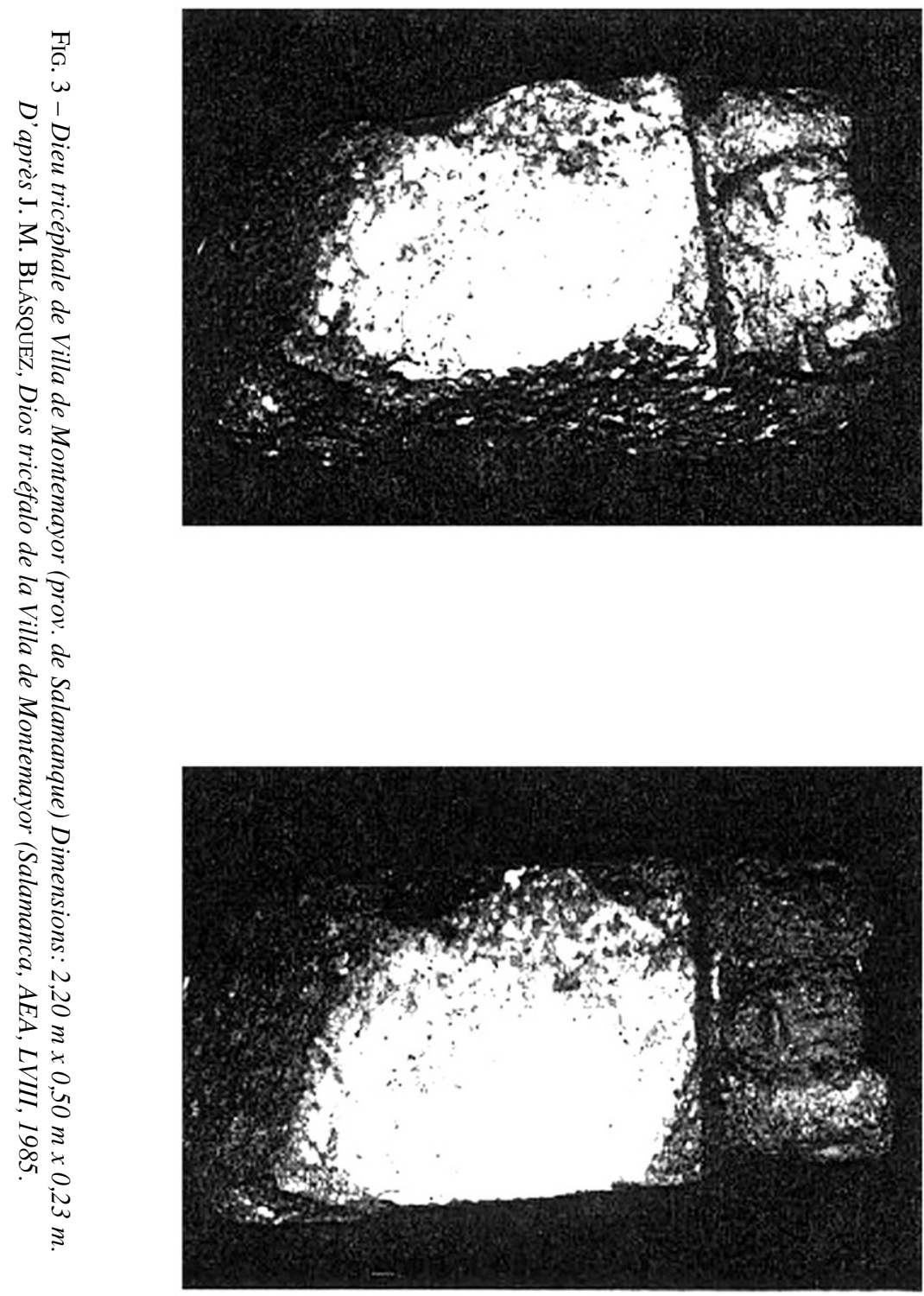


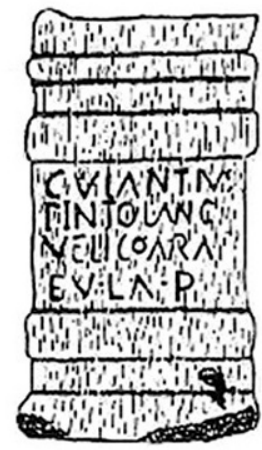

$0,51 \mathrm{~m} \times 0,27 \mathrm{~m} \times 0,20 \mathrm{~m}$

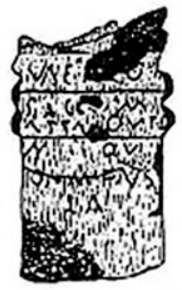

$0,34 \mathrm{~m} \times 0.20 \mathrm{~m} \times 0,15 \mathrm{~m}$

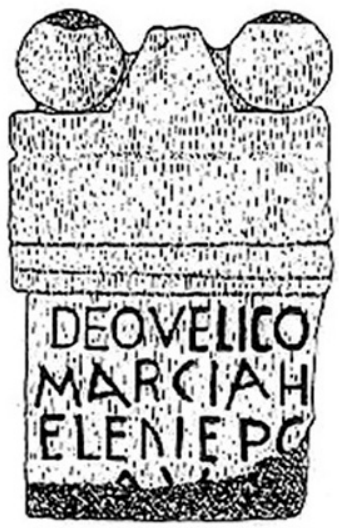

$0,58 \mathrm{~m} \times 0,38 \mathrm{~m} \times 0,30 \mathrm{~m}$

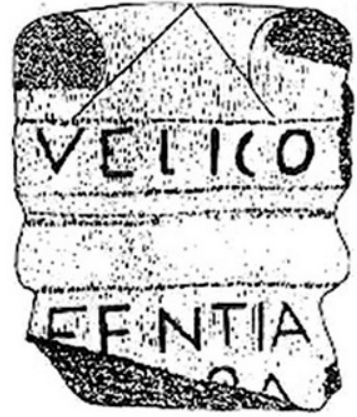

$0,20 \mathrm{~m} \times 0,17 \mathrm{~m} \times 0,13 \mathrm{~m}$

FIG. 4 - Autels de Postoloboso (prov. d'Avila) consacrés à Velicus

D’Après J. M. FERnÁNDEZ GóMEZ, Excavaciones arqueológicas en el Raso de Candeleda (Ávila) Ávila, 1986. 

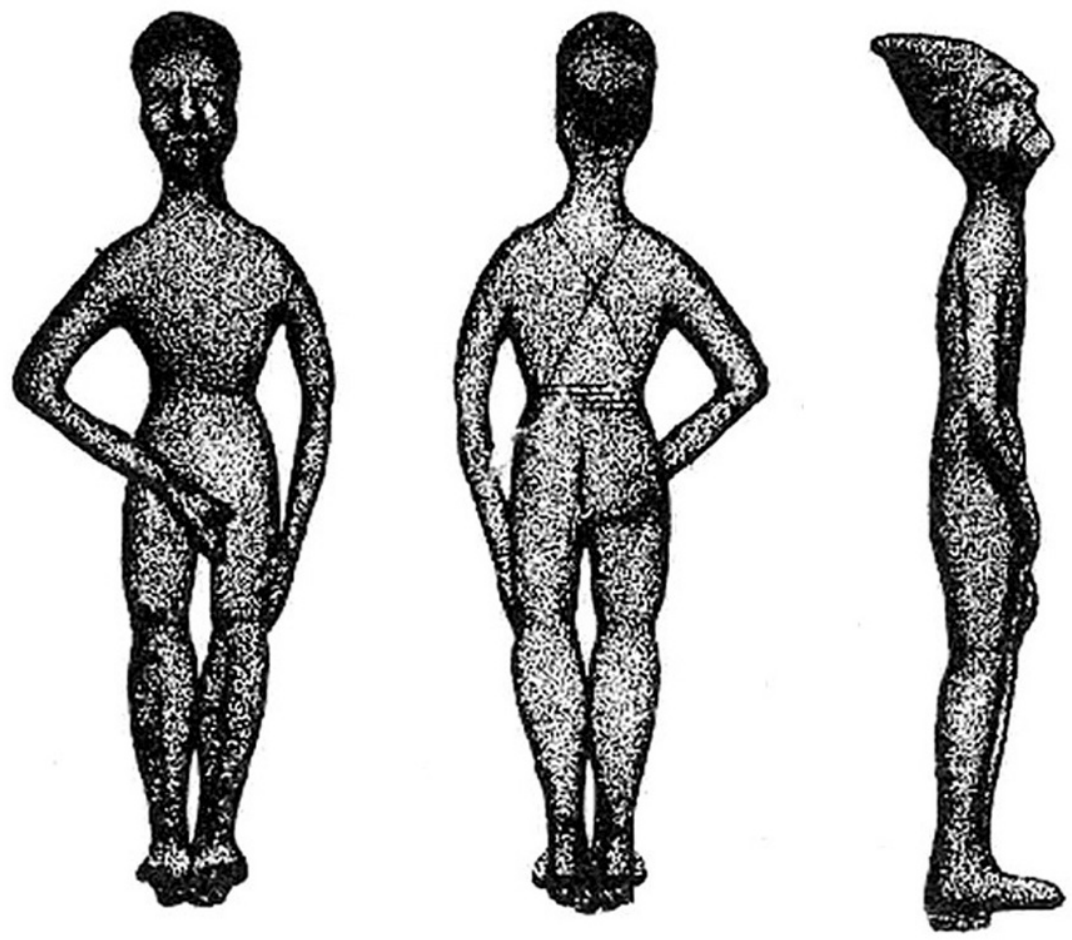

FIG. 5 - Statuette de Postoloboso

Hauteur. 10,8 cm

D’Après J. M. FERnÁNDEZ GómEZ, Excavaciones arqueológicas en el Raso de Candeleda (Ávila) Ávila, 1986. 

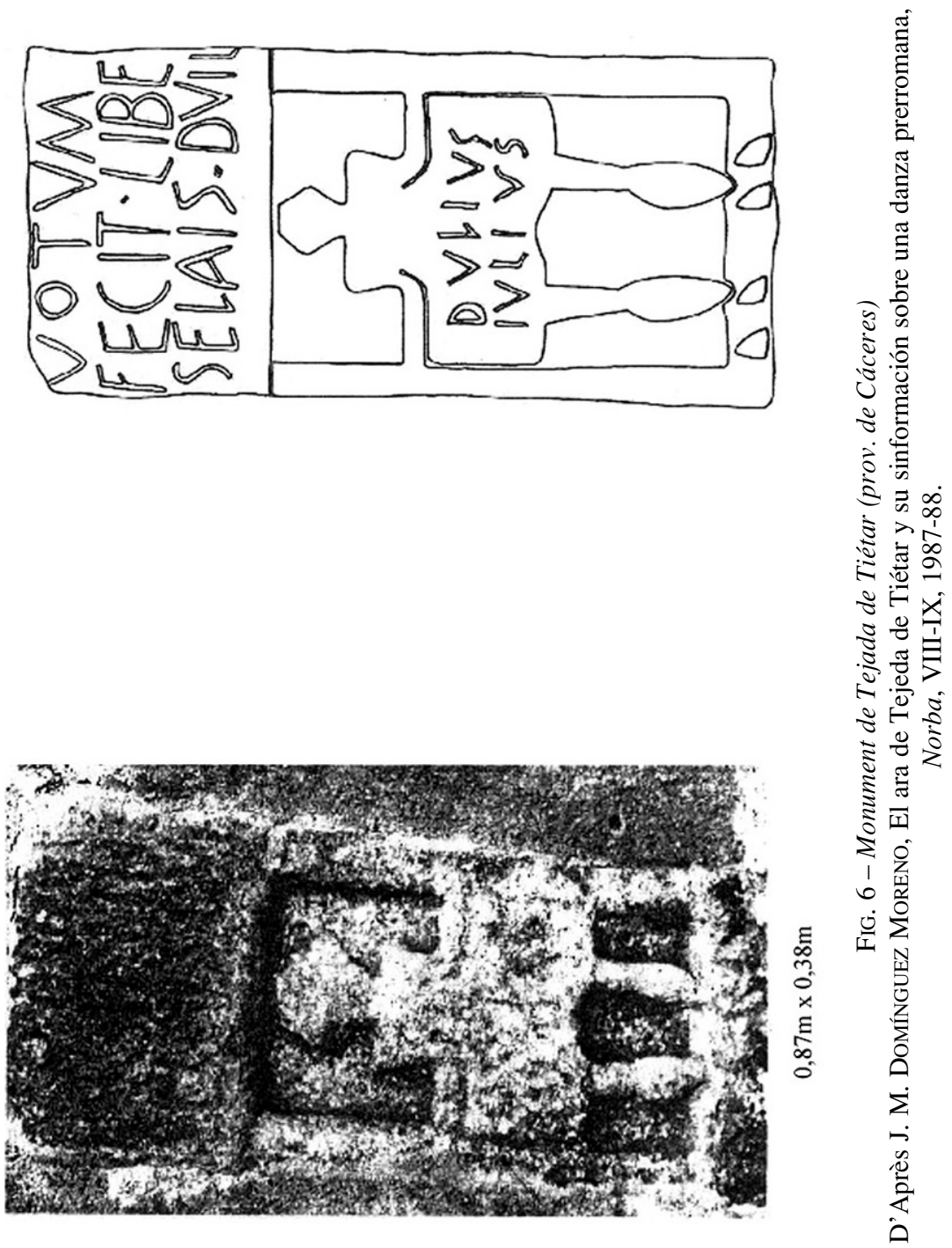


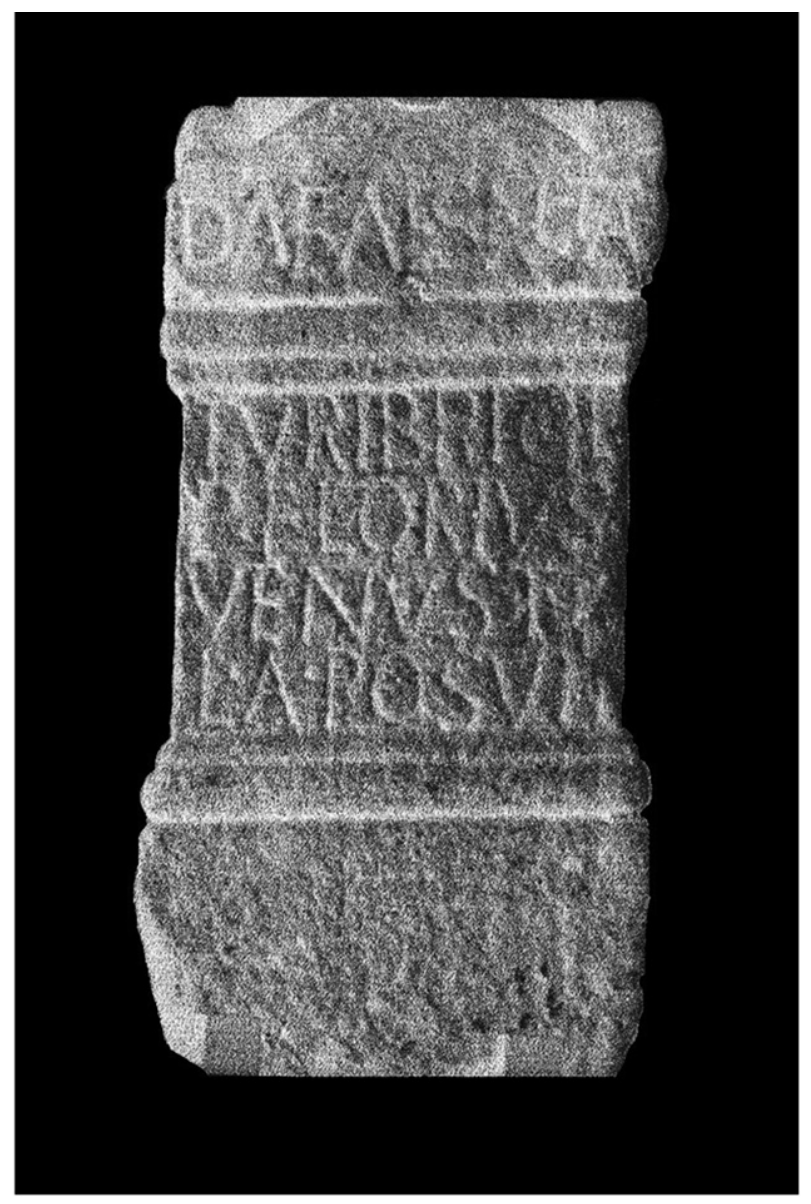

FIG. 7 - Autel dedié à Daedae Sanctae Turibrige(nsis) Herguijuela, prov. de Cáceres). Musée de Cáceres. 